\title{
hnRNP L-dependent protection of normal mRNAs from NMD subverts quality control in B cell lymphoma
}

\author{
Aparna Kishor, Zhiyun Ge \& J Robert Hogg* (iD
}

\begin{abstract}
The human nonsense-mediated mRNA decay pathway (NMD) performs quality control and regulatory functions within complex post-transcriptional regulatory networks. In addition to degradation-promoting factors, efficient and accurate detection of NMD substrates involves proteins that safeguard normal mRNAs. Here, we identify hnRNP $L$ as a factor that protects mRNAs with NMD-inducing features including long $3^{\prime} U T R s$. Using biochemical and transcriptome-wide approaches, we provide evidence that the susceptibility of a given transcript to NMD can be modulated by its $3^{\prime}$ UTR length and ability to recruit hnRNP L. Integrating these findings with the previously defined role of polypyrimidine tract binding protein 1 in NMD evasion enables enhanced prediction of transcript susceptibility to NMD. Unexpectedly, this system is subverted in B cell lymphomas harboring translocations that produce $B C L 2: I G H$ fusion mRNAs. CRISPR/Cas9 deletion of hnRNP $L$ binding sites near the BCL2 stop codon reduces expression of the fusion mRNAs and induces apoptosis. Together, our data indicate that protection by hnRNP $L$ overrides the presence of multiple $3^{\prime} U T R$ introns, allowing these aberrant mRNAs to evade NMD and promoting BCL2 overexpression and neoplasia.
\end{abstract}

Keywords B cell lymphoma; BCL2; hnRNP L; nonsense-mediated mRNA decay; UPF1

Subject Categories Cancer; RNA Biology

DOI 10.15252/embj.201899128 | Received 30 January 2018| Revised 17 October 2018| Accepted 25 October 2018| Published online 7 December 2018

The EMBO Journal (2019) 38: e99128

See also: MF Wilkinson \& H Cook-Andersen (February 2019)

\section{Introduction}

Nonsense-mediated decay (NMD) is a co-translational RNA decay mechanism that is evolutionarily conserved throughout eukaryotes (Nasif et al, 2018). NMD is named for its role in the decay of transcripts containing premature termination codons (PTCs) but is also involved in the decay of various classes of error-free mRNAs (Losson \& Lacroute, 1979; Maquat et al, 1981; Peltz et al, 1993). Examples of "normal" mRNAs targeted by NMD are those with upstream open reading frames (uORFs), exon-junction complexes (EJCs) downstream of a normal termination codon (TC), or long $3^{\prime}$ untranslated regions (3'UTRs; Rebbapragada \& Lykke-Andersen, 2009). Due to this broad substrate specificity, NMD has global effects on transcriptome stability through its function as both a surveillance mechanism and a regulatory pathway.

The mechanism for activation of the NMD machinery is still being elucidated and may vary by the type of transcript being targeted. In all cases, however, UPF1 is its central conductor, determining transcript stability by engaging in direct or indirect interactions with mRNAs, the translation machinery, EJCs, and decay enzymes. UPF1 is an RNA helicase that preferentially accumulates on $3^{\prime}$ UTRs through a combination of non-specific binding and displacement from coding sequences by elongating ribosomes (Hogg \& Goff, 2010; Hurt et al, 2013; Kurosaki \& Maquat, 2013; Zünd et al, 2013; Lee et al, 2015). As a result, UPF1 is enriched on long $3^{\prime}$ UTRs, where it has the potential to favor decay of transcripts with extended 3'UTRs (Hogg \& Goff, 2010). Interactions between UPF1 and the terminating ribosome, either directly or bridged by UPF3 proteins, are thought to promote UPF1 phosphorylation, which in turn recruits and activates the SMG6 endonuclease and general mRNA decay factors (Kashima et al, 2006; Huntzinger et al, 2008; Eberle et al, 2009; Okada-Katsuhata et al, 2012; Loh et al, 2013; Chakrabarti et al, 2014; Nicholson et al, 2014; Neu-Yilik et al, 2017).

In lower eukaryotes such as yeast, short (<200 nt), homogenous $3^{\prime}$ UTRs make surveillance of $3^{\prime}$ UTR length an effective strategy to identify PTCs (Jan et al, 2011; Celik et al, 2017). In contrast, mammalian mRNAs are subject to extensive post-transcriptional regulatory mechanisms that combine to determine their stability, translation efficiency, localization, and function. Much of this regulation is achieved via specific interactions between mRNA $3^{\prime}$ UTRs and trans-acting factors, including miRNAs and RNA-binding proteins (Mayr \& Bartel, 2009). The increased function of transcript $3^{\prime}$ UTRs has led to a broad distribution of vertebrate $3^{\prime}$ UTR lengths spanning the range from tens to thousands of nucleotides (Jan et al, 2011). To partially solve the problem of accurately detecting PTCs against a background of normal, long $3^{\prime}$ UTRs, vertebrates have 
evolved to use the exon-junction complex (EJC) as a marker for PTCs by linking the EJC to the NMD machinery (Le Hir et al, 2016). Yet vertebrates have also retained the ability to perform NMD in the absence of a downstream EJC, once again pointing to long $3^{\prime} \mathrm{UTR}$ as substrates for decay. This $3^{\prime}$ UTR EJC-independent decay has been observed to be triggered by diverse artificial 3'UTRs of $\sim 500 \mathrm{nt}$ or greater in human cells (Bühler et al, 2006; Matsuda et al, 2007; Singh et al, 2008; Hogg \& Goff, 2010; Baker \& Hogg, 2017), a length exceeded by thousands of physiological human $3^{\prime}$ UTRs.

How, then, is the pathway capable of deciding which long $3^{\prime} \mathrm{UTR}$ should be degraded and which should be allowed to persist? One possible strategy is to constrain detection of long $3^{\prime}$ UTRs by actively safeguarding pools of specific transcripts. Indeed, protection of genuine long $3^{\prime}$ UTRs has been found to be achieved by specific sequence elements found near termination codons in many transcripts (Chester et al, 2003; Weil \& Beemon, 2006; Toma et al, 2015; Ge et al, 2016). The phenomenon was most thoroughly described for Rous sarcoma virus transcripts in which the presence of an RNA stability element (RSE) prevents NMD-mediated destabilization (Weil \& Beemon, 2006; Weil et al, 2009; Withers \& Beemon, 2010; Hogg, 2016; Balagopal \& Beemon, 2017). In our previous work, we showed that the viral RSE is recognized and bound by the cellular protein polypyrimidine tract binding protein 1 (PTBP1), which confers protection from NMD when placed immediately downstream of a NMD-susceptible TC (Ge et al, 2016). Critically, cellular transcripts that can be similarly recognized by РTBP1 are also protected from NMD. Although the mechanism has yet to be fully elucidated, PTBP1 occupancy inhibits UPF1 accumulation on long 3'UTRs.

Leveraging our knowledge of PTBP1-mediated NMD inhibition, we here identify a role for hnRNP L in shielding normal transcripts from NMD. hnRNP L shares many functional and structural properties with PTBP1 but recognizes entirely distinct sequences (Zhang et al, 2013; Blatter et al, 2015), enabling the protection of a wider diversity of mRNAs. More broadly, we find that taking hnRNP L and PTBP1 binding potential into account significantly enhances the power of $3^{\prime}$ UTR length to predict UPF1 binding and NMD. Among the mRNAs that rely on hnRNP L for protection from NMD is BCL2, a potent antiapoptotic factor normally encoded by a transcript with an approximately 5,000-nt $3^{\prime}$ UTR. Our data indicate that the protection of BCL2 transcripts by hnRNP L extends to BCL2:IGH fusion transcripts, oncogenic mRNAs frequently produced by chromosomal translocations in follicular and diffuse large B cell lymphomas, despite the fact that they harbor multiple $3^{\prime}$ UTR introns. CRISPR/ Cas9-mediated deletion of hnRNP L binding sites in the aberrant fusion 3'UTR causes reduced BCL2 expression and increased apoptosis in a B cell lymphoma line. Together, these results explain global determinants of NMD target selection and uncover an unexpected failure of mRNA quality control important for the development of common B cell lymphomas.

\section{Results}

\section{PTBP1 and hnRNP L motifs predict reduced UPF1 association with mRNAs}

Our previous work demonstrated that a feature of the protective effect of PTBP1 in shielding transcripts from NMD is that PTBP1 binding near the TC excludes UPF1 binding (Ge et al, 2016). Here, we used RNA-binding protein (RBP) motif analysis in order to identify other proteins that may also modulate NMD efficiency by altering UPF1 occupancy on $3^{\prime}$ UTRs. Toward this goal, we identified the occurrences of TC-proximal motifs for several abundant RBPs transcriptome-wide and evaluated the relationship between motif density and transcript co-IP efficiency with UPF1 in an $\alpha$-UPF1 RIPSeq dataset generated by Lee et al (2015; Fig 1A). In this analysis, PTBP1 behaved in a manner consistent with our previous results: Transcripts with increased PTBP1 motif density in the first $400 \mathrm{nt}$ downstream of the TC were less efficiently recovered by UPF1 than mRNAs with a lower density. Of the other proteins analyzed, hnRNP L, hnRNP C, and hnRNP $\mathrm{U}$ demonstrated a similar pattern to PTBP1.

Many factors complicate interpretation of this analysis, including the possibility of overlapping RBP specificities and indirect effects on UPF1 binding due to differential localization, translation, or decay. Given these considerations, hnRNP L appeared to be the most promising as a bona fide protective protein among the three candidates due to its overall structural similarity to PTBP1 (Zhang et al, 2013; Blatter et al, 2015). The two proteins adapt a common protein architecture for differential RNA recognition, with PTBP1 interacting with sequences enriched in pyrimidines and hnRNP L preferring sequences high in CA repeats (Hui et al, 2003; Oberstrass et al, 2005; Martinez-Contreras et al, 2007). Further suggestive of similar roles for the proteins, we observed that the populations of transcripts with TC-proximal hnRNP L or PTBP1 motifs each had significantly longer $3^{\prime}$ UTRs than those without (Fig EV1A and B).

\section{hnRNP L binding near TCs correlates with NMD resistance}

We further assessed a potential role for hnRNP L in NMD substrate selection by analyzing hnRNP L enhanced crosslinking immunoprecipitation (eCLIP) and UPF1 knockdown RNA-seq datasets generated by the ENCODE Consortium (Sundararaman et al, 2016; Van Nostrand et al, 2016). Consistent with a protective function of hnRNP L, transcripts with hnRNP L CLIP peaks in the first $100 \mathrm{nt}$ of the $3^{\prime}$ UTR were significantly less sensitive to changes in cellular UPF1 levels than those lacking $3^{\prime}$ UTR peaks (Fig 1B; compare blue line to black line). Although protection was most prominent for transcripts containing peaks within $100 \mathrm{nt}$ of the TC, a significant protective effect was observed for transcripts containing $3^{\prime}$ UTR CLIP peaks outside of the first $400 \mathrm{nt}$ downstream of the TC only (Fig 1B; orange line). Importantly, transcripts with $5^{\prime} \mathrm{UTR}$ or coding sequence hnRNP L peaks but lacking 3'UTR peaks did not differentially respond to UPF1 knockdown, suggesting that hnRNP L binding events outside of the $3^{\prime}$ UTR do not influence NMD susceptibility (Fig 1B; purple line).

To evaluate positional effects of hnRNP L binding through the first 1,000 nt of the $3^{\prime} \mathrm{UTR}$, we focused on the set of transcripts with $3^{\prime}$ UTRs longer than $1 \mathrm{~kb}$ and assessed hnRNP L binding events in 100-nt segments. Transcripts with hnRNP L CLIP peaks in the first $100 \mathrm{nt}$ of the $3^{\prime} \mathrm{UTR}$ exhibited the greatest resistance to NMD, while having CLIP peaks in the remaining intervals was associated with an intermediate level of protection from NMD (Fig EV1C, colored circles). Because many transcripts contain CLIP peaks in multiple $3^{\prime}$ UTR intervals, we also evaluated the effect of removing transcripts 
A

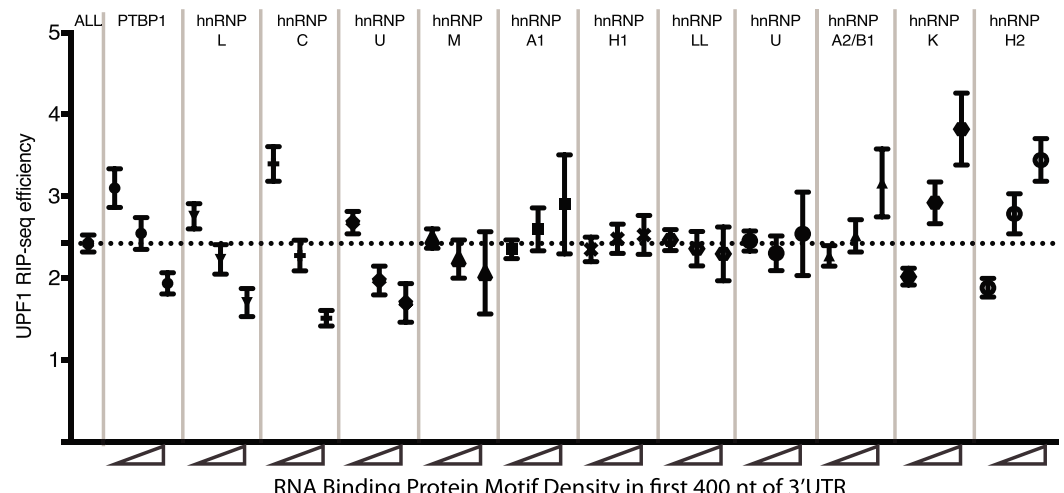

RNA Binding Protein Motif Density in first 400 nt of $3^{\prime}$ UTR
B

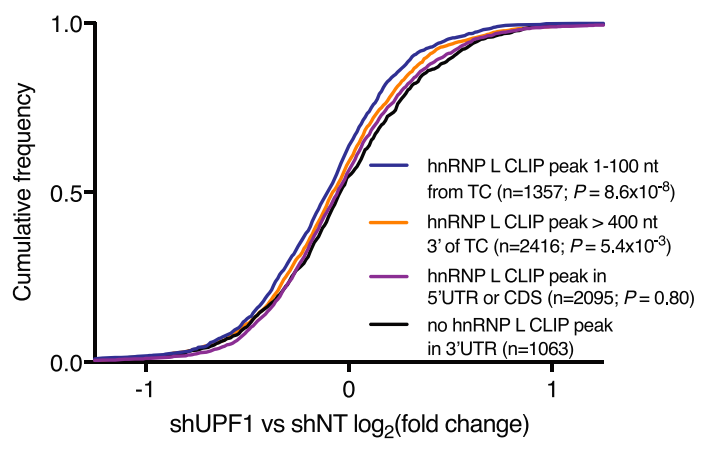

RSE
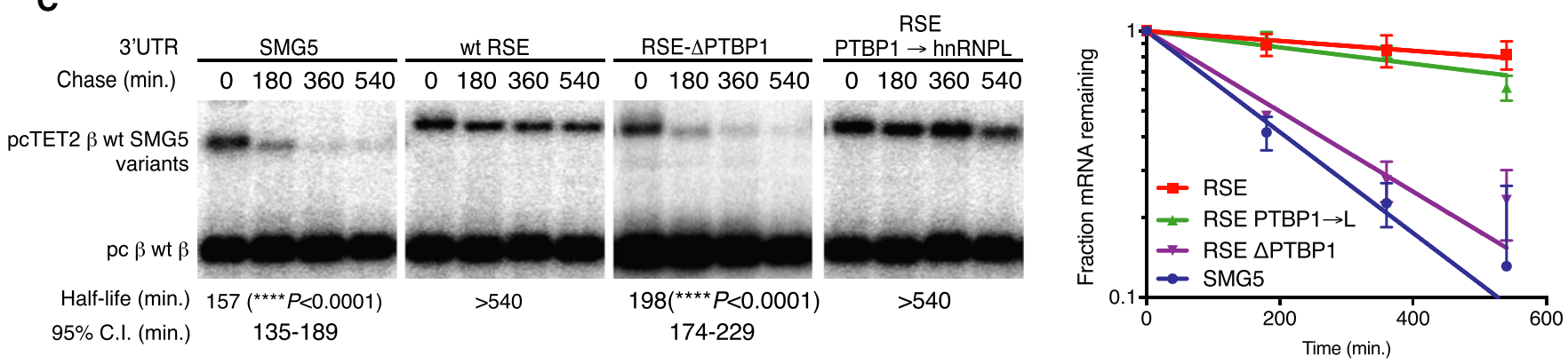

D

Bound
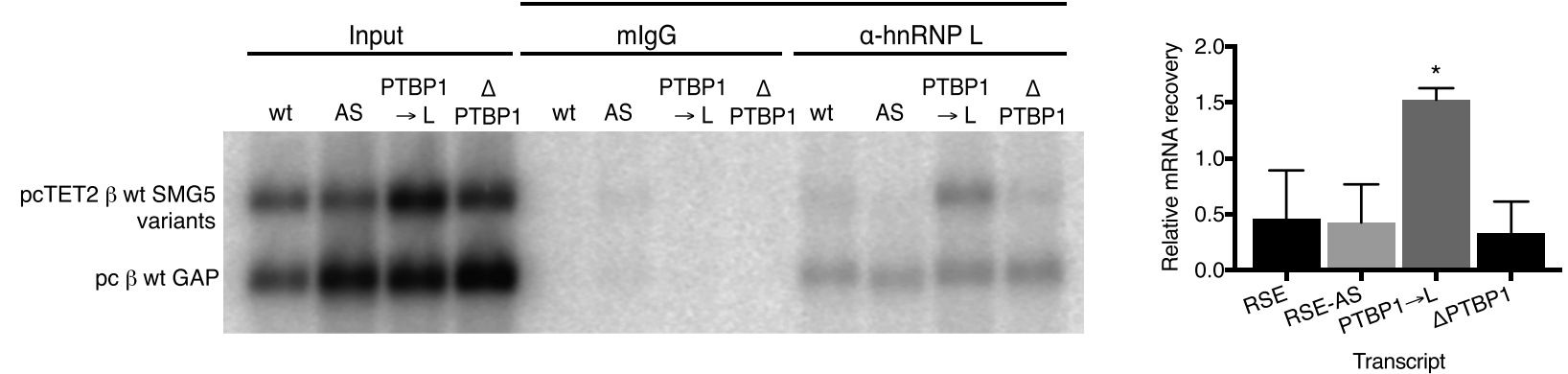

Figure 1. hnRNP L binds and stabilizes reporter transcripts.

A The first $400 \mathrm{nt}$ of all $3^{\prime}$ UTRs represented in the UPF1 RIP-seq dataset (Lee et al, 2015) was analyzed for the occurrence of motifs corresponding to the indicated RNAbinding proteins. Transcripts were divided into groups based on the number of motifs present $(0,1$, more than 1$)$, indicated by the gradient triangle. The dotted line indicates the mean RIP-seq efficiency for all transcripts used in the analysis. Mean and SD are plotted for all groups.

B CDF plot of relative abundance of transcripts with $3^{\prime} U$ TRs $>1,000$ nt in K562 cells under conditions of UPF1 or non-targeting shRNA, for transcripts with hnRNP L eCLIP peaks in the indicated intervals (Sundararaman et al, 2016). Each trace represents a category of CLIP peak locations. For mRNAs with peaks $>400 \mathrm{nt}$ from the TC or with peaks in the $5^{\prime} U T R$ or CDS, transcripts with peaks at $3^{\prime} U T R$ positions 1-400 were excluded. Statistical significance was determined by Kruskal-Wallis test with Dunn's correction for multiple comparisons and was determined based on comparison to the set with no 3'UTR CLIP peaks.

C Representative northern blots assaying the stability of $\beta$-globin reporter transcripts containing the SMC5 $3^{\prime} U T R$ alone or supplemented with cassettes containing the indicated RSE variants in HeLa Tet-Off cells. Half-lives were determined from at least three independent experiments, and decay curves are shown with points representing mean and standard deviation at each time point. Significance was measured compared to the SMC5 $3^{\prime}$ UTR reporter using the sum-of-squares $F$ test.

D RNA immunoprecipitation experiments evaluating hnRNP $L$ association with deletion and replacement reporter transcripts from (C). "AS" indicates a reporter in which the antisense sequence of the RSE was inserted in the SMG5 $3^{\prime}$ UTR in the same position as the RSE. The northern blot is representative of three independent experiments, and quantification is presented in the accompanying bar graph with mean and standard deviation. ${ }^{*} P=0.0146$ in two-tailed Student's $t$-test. The reporter containing the GAP $3^{\prime} U T R$, used here as a transfection control, contains a predicted hnRNP L binding site, allowing its consistent recovery in the IPS.

Source data are available online for this figure.

with peaks in the first $100 \mathrm{nt}$ of the $3^{\prime} \mathrm{UTR}$ from the analysis of peaks in the remaining intervals. Consistent with a central role for TCproximal binding events, the protection from NMD conferred by
hnRNP L binding in each 100-nt region was mitigated when the transcripts with peaks in the first 100 nucleotides were excluded (Fig EV1C, triangles). Conversely, exclusion of transcripts with 
peaks in any individual 100-nt interval from the set of mRNAs with peaks in the first $100 \mathrm{nt}$ did not affect the extent of protection observed (Fig EV1D). Together, these data reinforce the importance of hnRNP L binding in the vicinity of the terminating ribosome, but also indicate that additional hnRNP L binding outside of the TCproximal region can contribute to protection, in a manner that does not depend on precise positioning.

\section{hnRNP L can substitute for PTBP1 in an NMD-inhibitory element}

In order to test the putative protective function of hnRNP L, we turned to reporter mRNAs containing the NMD-inhibitory Rous sarcoma virus RSE. We previously showed that the RSE contains CU-rich motifs that bind cellular PTBP1, permitting viral transcripts to evade NMD (Ge et al, 2016). For the present investigation, we converted the PTBP1 binding sites in the RSE to hnRNP L binding sites by mutating uridines to adenosines. Wild-type and modified RSE variants were assayed for their ability to protect reporter mRNAs containing the well-characterized NMD target SMG5 3'UTR (Fig 1C; Singh et al, 2008; Yepiskoposyan et al, 2011; Ge et al, 2016). Decay assays comparing the half-lives of mRNAs in which RSE variants with PTBP1 or hnRNP L sites were placed upstream of the SMG5 3 'UTR exhibited equivalent transcript stability. In contrast, a construct with the PTBP1 motifs mutated to prevent recognition by either protein (RSE- $\triangle \mathrm{PTB}$ ) resulted in loss of the RSE stabilizing function (Ge et al, 2016). To verify that the CA-containing reporters were bound by hnRNP L, we assessed co-immunoprecipitation efficiency of the constructs with endogenous hnRNP L. As expected, hnRNP L preferentially co-purified reporter transcripts modified to contain hnRNP L binding motifs (Fig 1D). Together, these findings strongly suggest that hnRNP L is a second RNA-binding protein capable of conferring protection from NMD.

\section{hnRNP L protects select human transcripts with long $3^{\prime}$ UTRs from NMD}

To evaluate of the role of hnRNP $\mathrm{L}$ in protecting the human transcriptome from NMD, we performed RNA-seq on triplicate populations of HEK-293 Tet-Off (HEK-293TO) cells. We compared control siRNA knockdowns to three different RNAi conditions: hnRNP L depletion to identify dependent transcripts, UPF1 depletion to identify putative NMD targets, and simultaneous depletion of both hnRNP L and UPF1 in order to uncover transcripts sensitive to NMD in the absence of hnRNP L. We identified transcripts from 1,059 genes that significantly decreased in abundance upon hnRNP L knockdown, of which 374 were fully or partially rescued by concurrent depletion of UPF1 and hnRNP L (Fig 2A). Characteristics of the RNA-seq dataset are presented in Appendix Table S1.

Our model predicts that transcripts with long $3^{\prime} \mathrm{UTR}$ are particularly reliant on protection from NMD. Motif analysis indicated that hnRNP L binding sites are enriched on transcripts with longer $3^{\prime}$ UTRs (Fig EV1A), but to test this hypothesis more directly, we analyzed the distribution of $3^{\prime}$ UTR lengths for all mRNAs that met expression cutoffs, transcripts that decreased in abundance upon hnRNP L knockdown, and the sub-population of hnRNP L-dependent mRNAs that were rescued by concurrent UPF1 knockdown
A

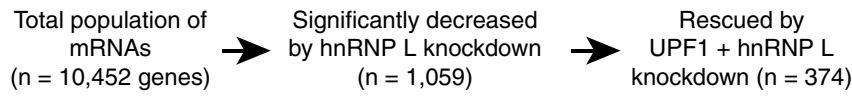

B
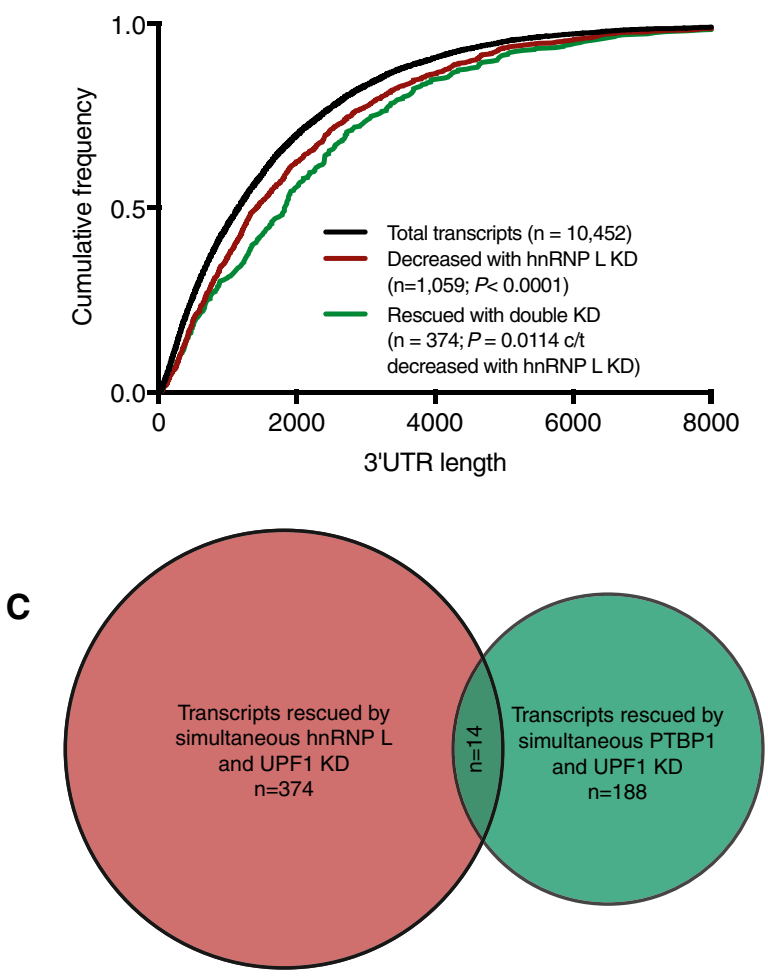

Figure 2. hnRNP L protects select human transcripts with long $3^{\prime}$ UTRs from NMD.

A RNA-seq analysis of cells treated with hnRNP $L$ siRNA or a combination of siRNAs against hnRNP $L$ and UPF1 identifies populations of transcripts that decreased in abundance upon hnRNP L knockdown in a UPF1-dependent manner.

B CDF plot of transcript 3'UTR lengths for subsets identified in (A). Statistical significance was determined by two-sided K-S test. $P$-value for decreased set is from comparison to the set of total transcripts. $P$-value for rescued set is from comparison to decreased set.

C Venn diagram (to scale) of populations of mRNAs putatively protected from NMD by hnRNP L or PTBP1.

Source data are available online for this figure.

(Fig 2B). For these analyses, we selected the most highly expressed transcript isoform from each gene, as judged by quantification with Kallisto software (Bray et al, 2016). Transcripts down-regulated by hnRNP L knockdown had significantly longer $3^{\prime}$ UTRs than the control population of mRNAs that was not reduced upon hnRNP L depletion, and mRNAs rescued in the double knockdown contained $3^{\prime}$ UTRs significantly longer than either of the other populations (Fig 2B).

Our RNA-seq data suggest that the populations of transcripts protected by PTBP1 or hnRNP L are largely distinct (Fig 2C), with the caveat that any transcripts protected by combined effects of the two proteins may not be detected by a single knockdown approach. As a result, we investigated the possibility that PTBP1 and hnRNP L 
execute parallel programs of NMD inhibition by conducting further transcriptome-wide motif analyses. Consistent with the observation that many mRNAs with hnRNP L CLIP peaks near the TC are also bound at one or more downstream sites, we find that high hnRNP L motif density proximal to the termination codon is correlated with higher hnRNP L and, to a lesser extent, PTBP1 motif densities in the remainder of the $3^{\prime} \mathrm{UTR}$ (Fig EV2A and B). Interestingly, transcripts with PTBP1 motifs near the TC are also much more likely to have additional 3'UTR PTBP1 sites than control mRNAs, but do not exhibit higher downstream hnRNP L motif density (Fig EV2C and D). Together, these findings suggest that TC-proximal hnRNP L and PTBP1 binding may be frequently accompanied by additional sites of recruitment across the $3^{\prime}$ UTR.

\section{hnRNP L confers variable degrees of protection from NMD}

We performed qRT-PCR on an independent set of knockdown experiments to examine the steady state levels of a panel of transcripts that were found to be decreased with cellular hnRNP L depletion in a UPF1-dependent manner in RNA-seq experiments (Fig 3A). Among these, we observed many, such as CSRP1 and BCL2, for which UPF1 knockdown alone did not significantly alter the abundance of the transcript. Hence, these transcripts appear to be completely protected from NMD in the presence of hnRNP L but sensitized to the pathway upon hnRNP L depletion. In addition, we observed a class of transcripts that depended on hnRNP $\mathrm{L}$ for normal accumulation and yet increased in abundance with UPF1 knockdown alone (e.g., PVRL4; Fig 3A). These transcripts may be partially protected from NMD by hnRNP L or may normally be subject to NMD and also become sensitive to additional regulatory mechanisms in the absence of hnRNP L. Assays of stability of selected transcripts by pulse-labeling with 5-ethynyl uridine (5-EU) confirmed that these mRNAs were destabilized when hnRNP L was knocked down (Fig 3B), including three transcripts (BCL2, CSRP1, and CUL7) that appear to be fully protected and one (PVRL4) that shows evidence of partial protection (Fig 3A).

To investigate the role of hnRNP L binding sites in establishing full or partial protection from NMD, we selected the CSRP1 $3^{\prime}$ UTR as a test case. Based on the qPCR data in HEK-293TO cells, CSRP1 appears to be completely protected. Its $3^{\prime} \mathrm{UTR}$ contains two regions with a high density of putative hnRNP L binding sites: the first 200 nt downstream of the TC and the last $200 \mathrm{nt}$ immediately prior to the polyadenylation signal. We determined the half-life of a panel of $\beta$-globin reporter transcripts with variant CSRP1 3'UTRs in which increasing tracts of hnRNP L motifs were mutated to decrease binding potential (Fig $3 \mathrm{C}$ and Appendix Fig S1). Consistent with findings from the CLIP dataset indicating that multiple binding events may together direct protection, mutation of hnRNP L binding sites in the first 30,100 , and $200 \mathrm{nt}$ of the $3^{\prime} \mathrm{UTR}$ partially and progressively destabilized the reporter mRNAs (Fig 3C). Mutation of motifs at the distal end of the 3'UTR (Fig 3C; nt 961-1,161) did not cause instability, once again highlighting the importance of hnRNP L binding proximal to the TC, as previously observed for PTBP1 (Ge et al, 2016). Confirming that the enhanced decay of the reporter was a result of NMD, UPF1 knockdown restored stability of the variant lacking hnRNP L binding sites in the first $200 \mathrm{nt}$ of the CSRP1 3'UTR (Fig 3D).

\section{hnRNP L motif density is inversely correlated with UPF1 binding and NMD}

The findings from the eCLIP analysis and CSRP1 reporter data relating to hnRNP L binding site position and density prompted us to take a unified approach to examine transcriptome-wide relationships among hnRNP L motif frequency, $3^{\prime}$ UTR length, and NMD through the lens of UPF1 RIP-seq enrichment. Motif analysis, unlike eCLIP, does not depend on transcript expression level in any particular cell type and may provide a general tool for analysis of mRNA susceptibility to NMD. Because hnRNP L eCLIP suggested an auxiliary role for hnRNP L binding at multiple sites in $3^{\prime}$ UTRs (Figs 1B, and EV1C and D), and both eCLIP and motif analyses revealed that TC-proximal protective protein association is often accompanied by potential for additional binding at multiple $3^{\prime}$ UTR sites (Figs EV1C and D, and EV2A and B), we used hnRNP L motif density throughout $3^{\prime}$ UTRs to categorize transcripts for this investigation.

We first asked whether efficient transcript recovery in UPF1 RIPSeq is predictive of NMD sensitivity, finding that transcripts that increased in abundance upon UPF1 knockdown in our RNA-seq data were indeed preferentially enriched in UPF1 RIP-seq relative to non-NMD substrates (Fig EV2E; Lee et al, 2015). With this confirmation, we divided the set of all cellular transcripts into bins on the

Figure 3. hnRNP L confers a spectrum of protection from NMD.

A GPCR of selected transcripts found by RNA-seq to decrease in abundance in the absence of hnRNP L. Values represent the mean and standard deviation of three independent biological replicates. Significance of hnRNP L KD and UPF1 KD was compared to NT, and the double knockdown was compared to hnRNP L KD. For clarity, only a single star is used to indicate significance; in all cases, $P \leq 0.025$ in two-tailed Student's $t$-tests.

B Half-lives of selected transcripts under conditions of non-targeting or hnRNP $L$ knockdown. Measurements were calculated based on proportional recovery of transcript labeled with 5 -EU in 1 h versus total transcript. Significance was determined using two-tailed Student's $t$-test: ${ }^{\star} P \leq 0.05$, ${ }^{\star \star} P \leq 0.01$, ${ }^{\star \star \star \star} P \leq 0.0001$. Values represent mean and standard deviation of three independent replicates.

C The stability of $\beta$-globin reporters with the CSRP1 $3^{\prime} U T R$, along with several variants in which putative hnRNP L motifs in the indicated intervals were mutated, was evaluated in HeLa Tet-Off cells. The schematic indicates areas of $3^{\prime}$ UTR CA enrichment in light blue and includes the sequence of the first 200 nucleotides of the CSRP1 $3^{\prime}$ UTR, where putative hnRNP L binding elements are in red typeface. The northern blot is a representative example from three independent replicates. Mean values plotted with SD at each time point were used to quantify decay (right panel). Significance is measured compared to the WT reporter using the sum-of-squares $F$ test.

D The stability of the WT and L mut 1-200 CSRP1 reporters in HeLa Tet-Off cells treated with non-targeting RNAi or siRNA against UPF1 was evaluated. The northern blot is a representative example from at least three biological replicates. Decay quantification and statistics were performed as in (C), with significance measured compared to the non-targeting condition.

Source data are available online for this figure. 
A

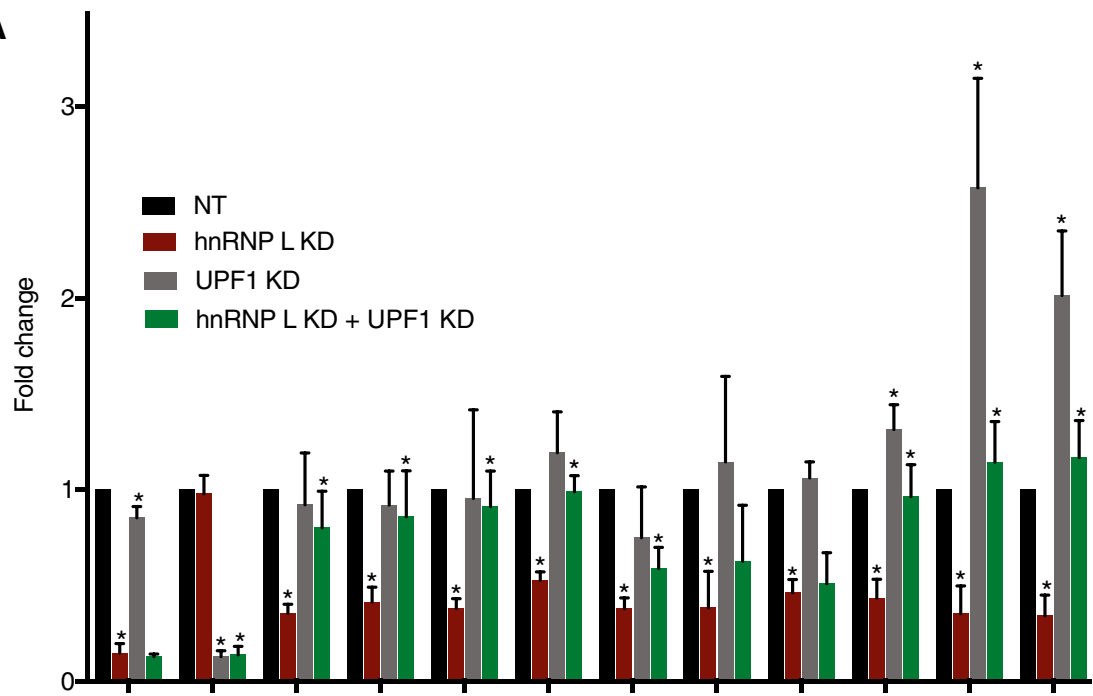

B

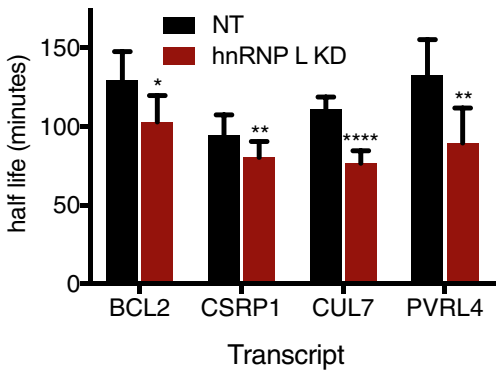

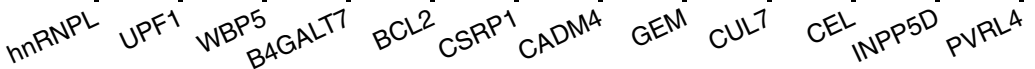

Transcript

C

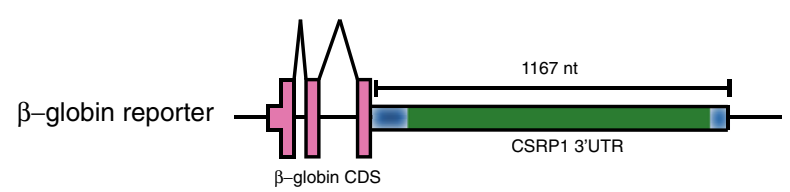

GGCCACCATCACCCACCACACCCTGCCCACTCCTGCGCTTTTCATCGCCATTCCATTCCCAGCAGCTTTGGAGACCTCCA GGATTATTTCTCTGTCAGCCCTGCCACATATCACTAATGACTTGAACTTGGGCATCTGGCTCCCTTTGGTTTGGGGGTCT GCCTGAGGTCCCACCCCACTAAAGGGCTCCCCAGGCCTGG
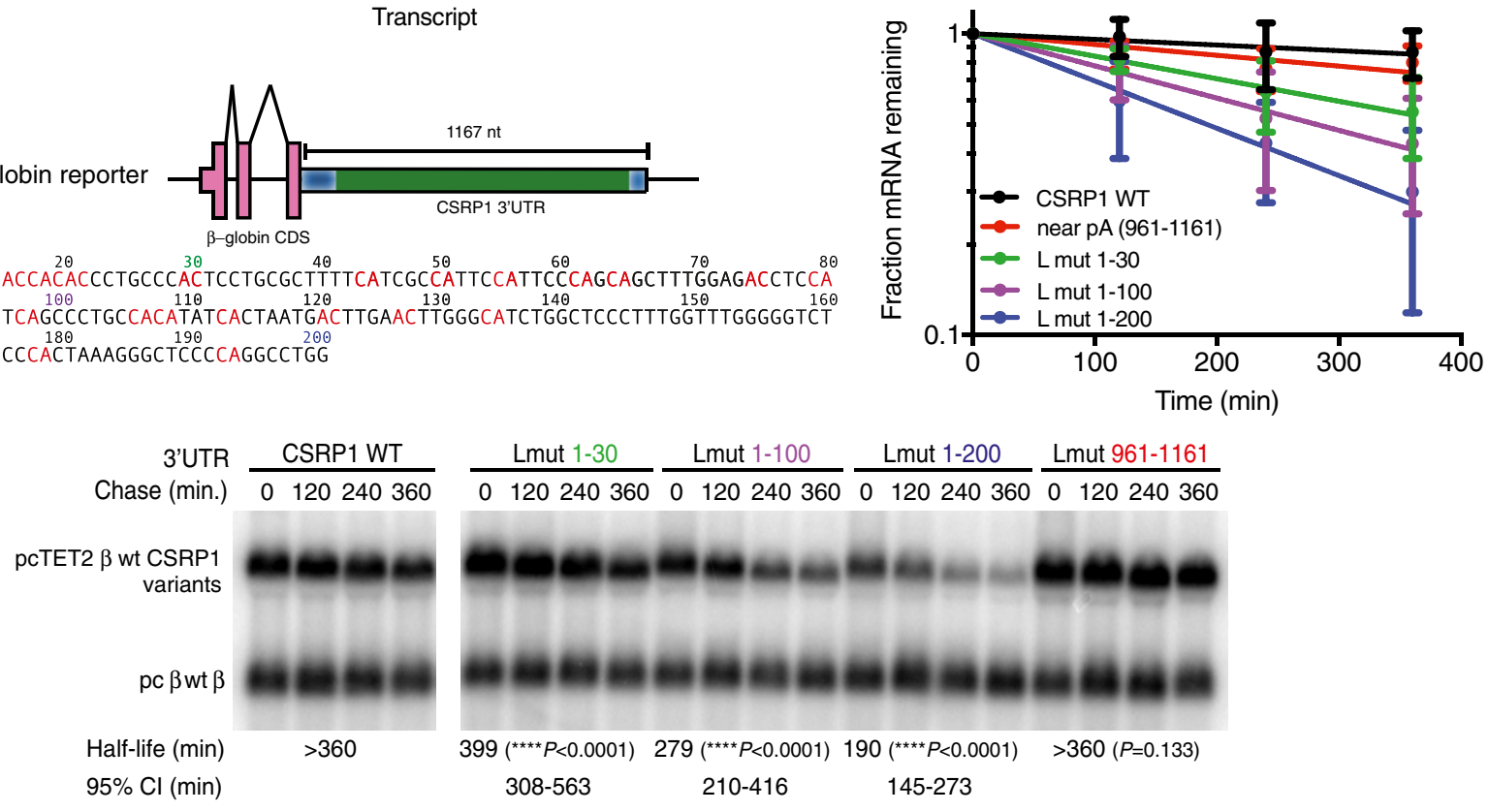

\section{D}
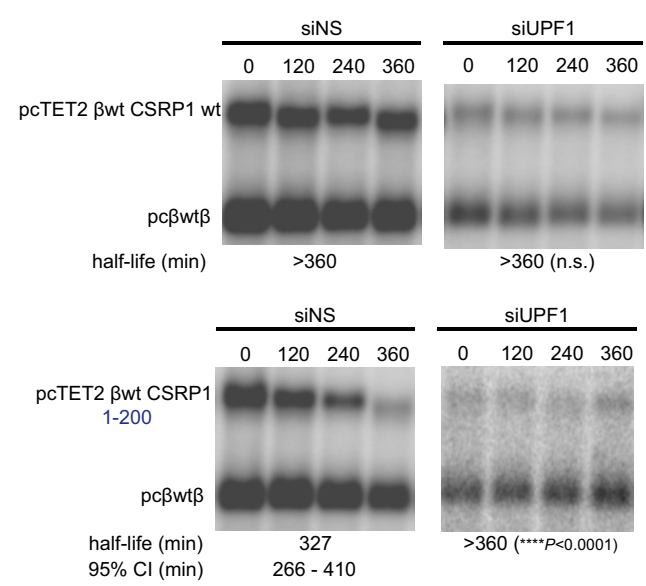
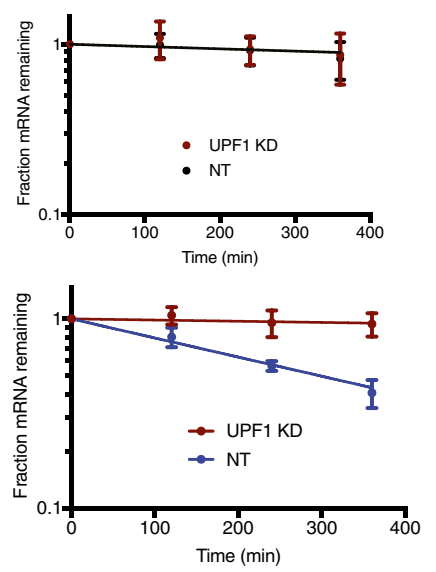

Figure 3. 
basis of $3^{\prime}$ UTR length (first tertile $<718 \mathrm{nt}$, second tertile $=718$ $1,885 \mathrm{nt}$, and third tertile $>1,885 \mathrm{nt}$ ) and hnRNP L motif density. Across the two longest $3^{\prime}$ UTR length classes, transcripts with moderate or high propensity to bind hnRNP L were significantly less efficiently recovered with UPF1 than those with low hnRNP L motif density (Fig 4A). Interestingly, high hnRNP L motif density was associated with significant reductions in UPF1 binding even among the shortest $3^{\prime}$ UTR class. Consistent with these findings from the UPF1 RIP-seq data, increased hnRNP L motif density correlated with an attenuated response to UPF1 depletion in RNA-seq: mRNAs with long $3^{\prime}$ UTRs and low hnRNP L motif density were significantly more likely to increase in abundance upon UPF1 knockdown than mRNAs with high hnRNP L motif density (Fig 4B). Together, these data are consistent with a model in which hnRNP L acts at an early stage of target discrimination by decreasing UPF1 binding to 3'UTRs.

\section{hnRNP L and PTBP1 motifs improve the power of $3^{\prime}$ UTR length to predict UPF1 binding and decay}

Since our studies suggest that PTBP1 and hnRNP L have complementary roles in shaping NMD target specificity, we asked whether combined analysis of PTBP1 and hnRNP L motifs results in more accurate prediction of transcript recognition by UPF1 and NMD. Classifying mRNAs by $3^{\prime}$ UTR length as above, we subdivided mRNAs based on the density of motifs for both PTBP1 and hnRNP L. Notably, mRNAs with $3^{\prime}$ UTRs in intermediate and long size classes with high PTBP1 and/or hnRNP L motif density exhibited
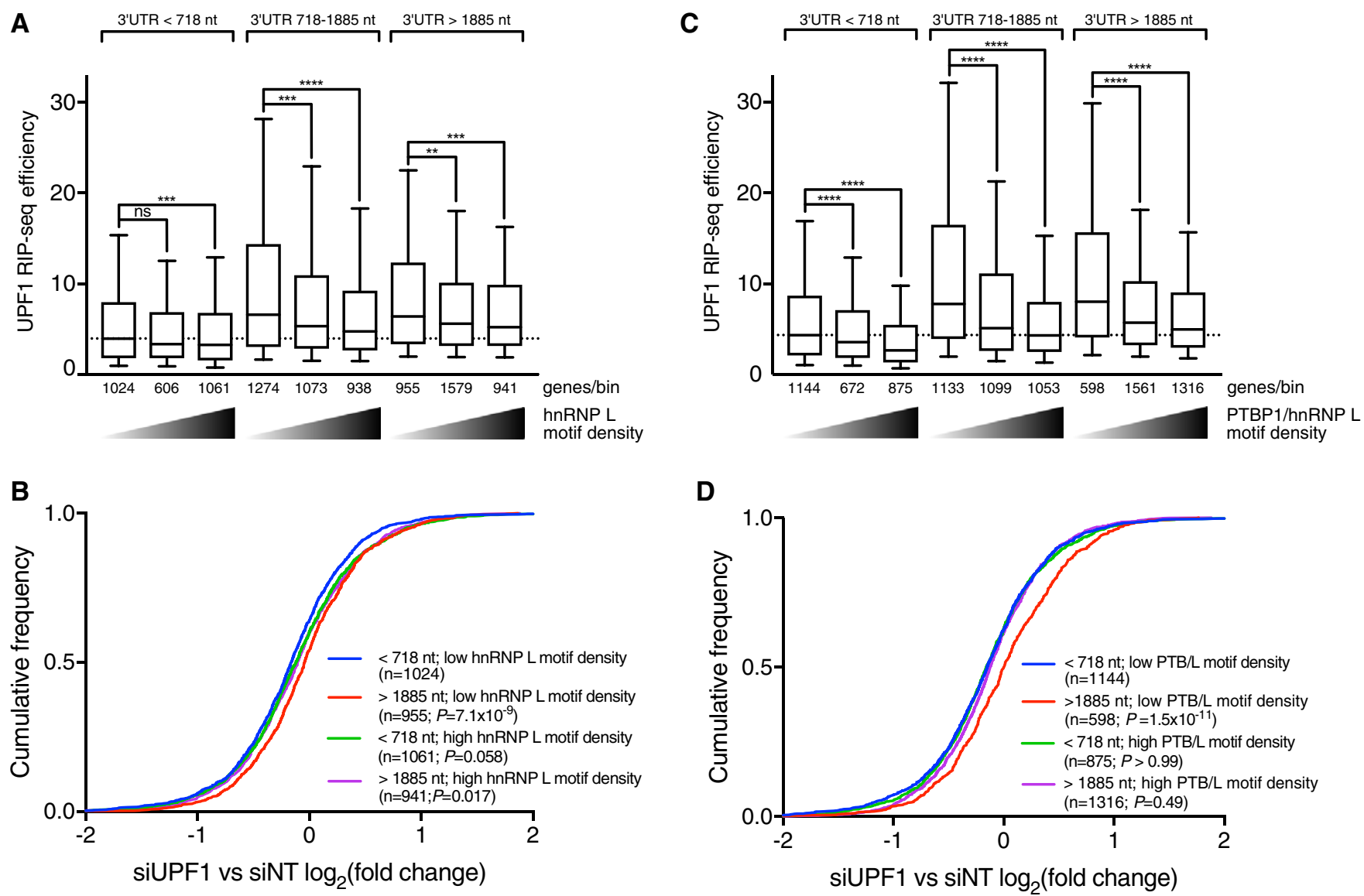

Figure 4. hnRNP L and PTBP1 motifs enhance predictive utility of $3^{\prime} U T R$ length.

A Motif analysis of recovered UPF1-associated transcripts analyzed by RIP-seq (Lee et al, 2015). Transcripts were binned into three $3^{\prime}$ UTR length classes, which were then further subdivided based on hnRNP L motif density. Each box plot represents the median and interquartile range with extensions from 10 to $90 \%$ of the indicated subset. Dotted line represents the median RIP-seq efficiency of the class of transcripts with the shortest $3^{\prime} U T R$ and no hnRNP L binding motifs. Significance was computed using Kruskal-Wallis tests with Dunn's correction for multiple comparisons.

B CDF plots of selected transcript classes from (A). $\log _{2}(F C)$ of UPF1 knockdown as compared to the non-targeting control indicates NMD sensitivity of each class. Significance was determined by Kruskal-Wallis test with Dunn's correction for multiple comparisons

C Motif analysis of recovered UPF1-associated transcripts analyzed by RIP-seq (Lee et al, 2015). Transcripts are divided into tertiles by hnRNP $L$ and PTBP1 binding motif density and $3^{\prime}$ UTR length. Data are represented and analyzed as in (A). Dotted line represents the median RIP-seq efficiency of the class of transcripts with the shortest $3^{\prime}$ UTRs and lowest PTBP1/hnRNP L motif density.

D CDF plots of selected transcript classes from (C) analyzed as in (B).

Data information: ${ }^{\star \star} P \leq 0.01,{ }^{* \star *} P \leq 0.001,{ }^{\star \star * *} P \leq 0.0001$. 
UPF1 binding similar to that observed among short 3'UTRs lacking binding sites (Fig 4C). Moreover, long $3^{\prime}$ UTRs with high PTBP1/ hnRNP L motif density showed no significant difference in response to UPF1 knockdown in comparison with mRNAs with short $3^{\prime} \mathrm{UTR}$ and low motif density (Fig 4D). In contrast, long 3'UTRs with low motif density were much more likely to increase in abundance upon UPF1 knockdown. In other words, transcripts with high hnRNP L and PTBP1 motif density did not show 3'UTR length-dependent sensitivity to UPF1; conversely, transcripts with low PTBP1 and hnRNP L binding potential showed a heightened correlation between $3^{\prime}$ UTR length and NMD (Fig EV3). Together, these data suggest that accurate prediction of UPF1 binding and NMD susceptibility based on $3^{\prime} \mathrm{UTR}$ length is significantly impaired because many transcripts are protected by PTBP1 and/or hnRNP L. Thus, incorporation of analyses of the binding potential of protective proteins can enhance predictions of which long $3^{\prime}$ UTRs will direct decay.

\section{hnRNP L protects the oncogenic BCL2:IGH fusion mRNAs from NMD}

BCL2 mRNAs were among those identified by RNA-seq and qPCR as completely protected by hnRNP L (Fig 3A). BCL2 has a long 3'UTR of approximately 5,000 nt, the first $200 \mathrm{nt}$ of which includes an extensive tract of CA repeats, making this transcript an excellent candidate for direct protection by hnRNP L (Fig 5A). Intriguingly, the $\mathrm{CA}$ tracts are highly conserved from rodents to humans (Appendix Fig S2). Previous work showed that hnRNP L binds the BCL2 transcript through these TC-proximal CA-rich sequences but did not address a possible role in NMD evasion (Lee et al, 2009).

Aberrant BCL2 expression is common in B cell lymphomas: Up to $90 \%$ of follicular and $20 \%$ of diffuse large B cell lymphomas carry reciprocal translocations between chromosomes 14 and 18 (Leich et al, 2011; Schuetz et al, 2012). Moreover, over $60 \%$ of healthy adult donors carry detectable circulating $\mathrm{B}$ cells that have undergone $t(14: 18)$ translocation and represent potential lymphoma precursors (Schüler et al, 2009). Approximately $50 \%$ of $t(14: 18)$ translocations associated with B cell lymphoma occur in the BCL2 major breakpoint region (MBR), fusing a portion of the IGH locus on chromosome 14 to the last exon of BCL2 on chromosome 18 and causing BCL2 transcriptional activation by the IGH $\mu$ enhancer (Cleary et al, 1986; Seto, 2002; Deng et al, 2007). At the mRNA level, MBR translocations preserve the BCL2 ORF, TC, and proximal $\sim 2,500 \mathrm{nt}$ of its $3^{\prime} \mathrm{UTR}$ (including protective hnRNP L binding sites), but the distal half of the BCL2 $3^{\prime}$ UTR is replaced by 4-6 IGH exons, depending on which IGH polyadenylation signal is used (Fig 5B; Cleary et al, 1986; Lim et al, 2010). These exons are efficiently spliced and would be expected to lead to EJC deposition and NMD. However, this putative NMD sensitivity is at odds with the high levels of BCL2 expression from the fusion chromosome known to be essential for cell survival and lymphomagenesis.

As the wild-type BCL2 transcript showed evidence of complete protection in $293 \mathrm{~T}$ cells (Fig 3A), we explored whether the aberrant BCL2 transcript is also able to use hnRNP L to evade NMD. To test this hypothesis, we used the well-characterized B cell lymphoma cell line SU-DHL-4, which carries $t(14 ; 18)$ at the BCL2 MBR as described above (Cleary et al, 1986). In these cells, the IGH $\mu$ enhancer drives high levels of transcription of the BCL2:IGH fusion, with minimal transcription of the BCL2 locus on the unaltered chromosome 18 (Cleary et al, 1986). To test the sensitivity of the BCL2: IGH fusion mRNA to NMD in this background, we treated cells with cycloheximide with and without RNAi-mediated knockdown of hnRNP L. In control knockdown cells, RT-PCR revealed that abundance of known NMD target mRNAs such as UPF1, GADD45B, NAT9, and TBL2 increased sharply after cycloheximide treatment (Fig 5C). Levels of the fusion mRNA increased just 1.3-fold with cycloheximide, indicating that the aberrant BCL2:IGH mRNAs may be only slightly sensitive to NMD or other translation-dependent regulation in this cell line. The abundance of fusion mRNA decreased upon hnRNP L depletion, an effect that was entirely reversed by cycloheximide treatment, results consistent with NMD resistance conferred by hnRNP L. Together, these findings suggest that BCL2 fusion mRNAs are largely protected from quality control by NMD.

\section{hnRNP L binding sites are required for protection of $B C L 2: I G H$ fusion transcripts}

Our data indicate that hnRNP L protection of the BCL2:IGH fusion transcript in SU-DHL-4 is robust despite multiple potential NMDinducing features. To directly interrogate the effects of hnRNP L binding and NMD on the BCL2 fusion transcript, we designed a family of reporter constructs to recapitulate the BCL2:IGH mRNA $3^{\prime}$ UTR splicing events and EJC placement in SU-DHL-4 cells (Fig 5D). The BCL2 fusion $3^{\prime}$ UTR containing four introns was cloned downstream of the $\beta$-globin CDS (Fusion), and variants with the hnRNP L motifs mutated to GU (Fus mut) or deleted entirely (Fus $\Delta$ ) were created to test the importance of hnRNP L recruitment (Appendix Fig S3). UPF1 knockdown caused a small increase in the half-life of the Fusion reporter, suggesting that a minor fraction of the reporter mRNAs can undergo NMD in this context (Fig 5E), consistent with the findings from cycloheximide treatment of SU-DHL-4 cells. As expected based on our knockdown studies, the Fusion reporter was significantly more stable than either variant lacking TC-proximal hnRNP L binding sites (the Fusion reporter had $t_{1 / 2}$ of 394 min compared to $t_{1 / 2}$ of $140 \mathrm{~min}$ for Fus $\Delta$ and $t_{1 / 2}$ of 187 min for Fus mut). Importantly, both of the variant reporters were highly stabilized with UPF1 knockdown, indicating that loss of hnRNP L binding sensitizes these reporters to clearance by NMD. These results suggest that the fusion BCL2 mRNAs in SU-DHL-4 cells are protected by hnRNP L binding sites near the TC.

\section{hnRNP L promotes survival of lymphoma cells with $t(14 ; 18)$}

We next tested whether hnRNP L is important for the survival of B cell lymphoma cell lines carrying the $t(14: 18)$ translocation at the BCL2 MBR. We first evaluated apoptosis in SU-DHL-4 cells in which hnRNP L was transiently depleted using RNAi. It has been shown that SU-DHL-4 cells are resistant to the high-affinity BCL2 inhibitor ABT-199 (marketed as venetoclax) due to the parallel activity of a second BCL2 family member, MCL1 (Souers et al, 2013; Li et al, 2015; Klanova et al, 2016; Phillips et al, 2016). In those studies, reduction of MCL1 activity with the MCL1 inhibitor A-1210477 in combination with targeted BCL2 inhibition with venetoclax was required to decrease cell viability. 
A

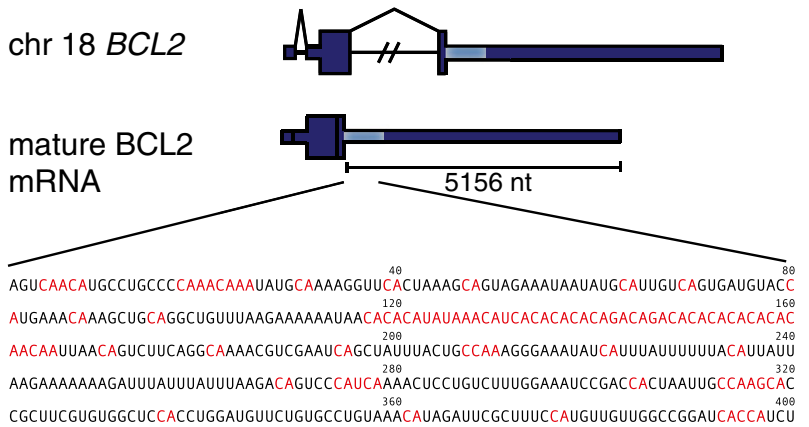

B

$t(14 ; 18)$

$$
\text { chr } 18 \text { BCL2 } \quad \operatorname{chr} 14 I G H
$$
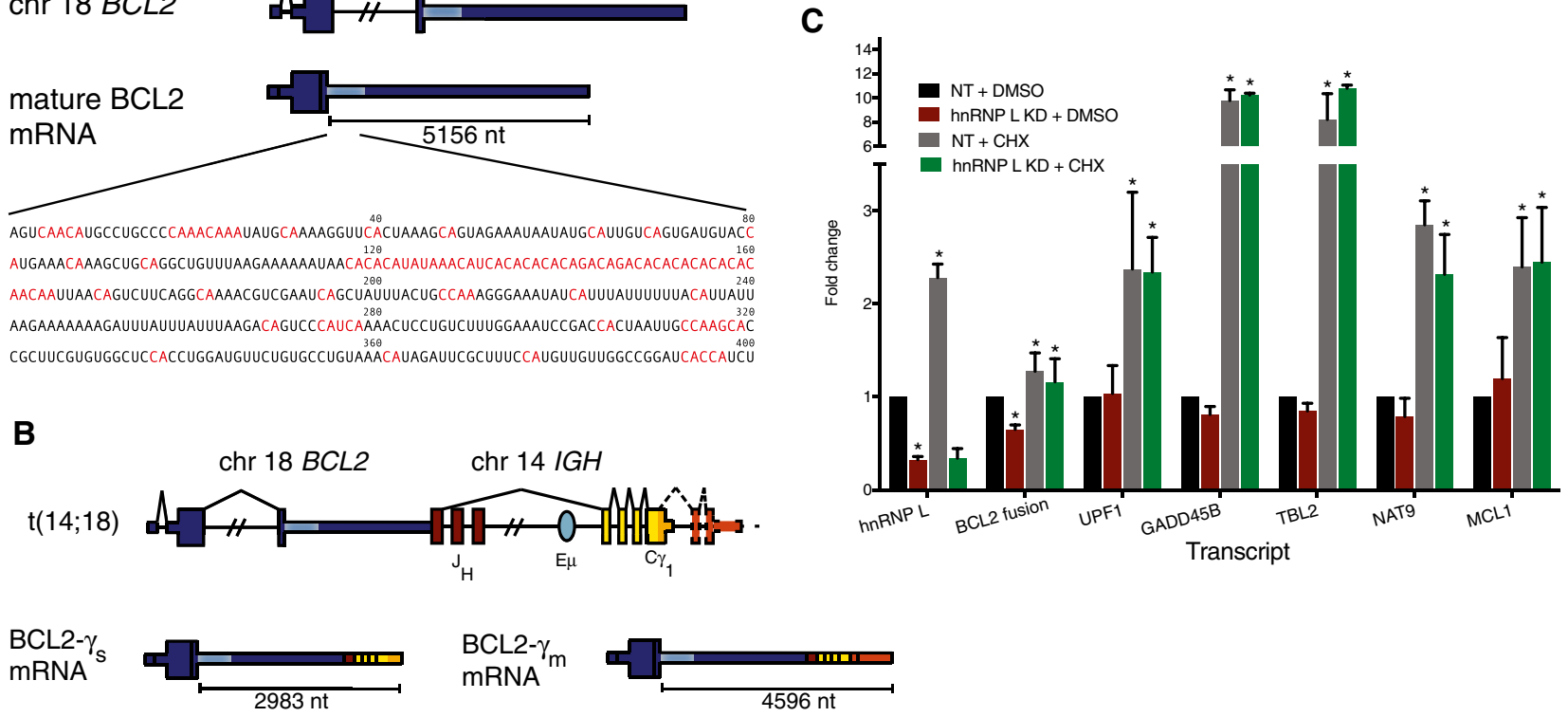

D

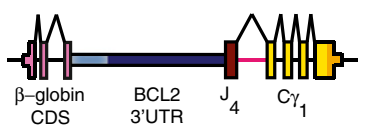

E

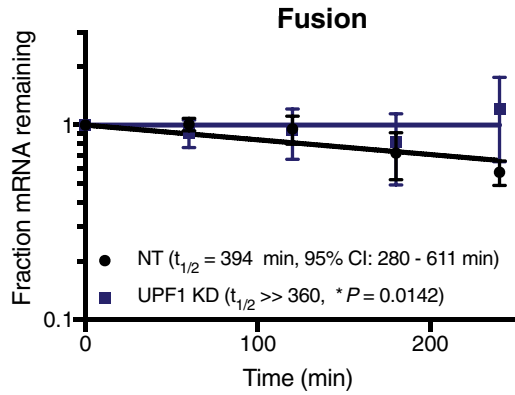

Fus $\Delta$

reporter mRNA

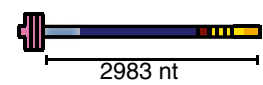

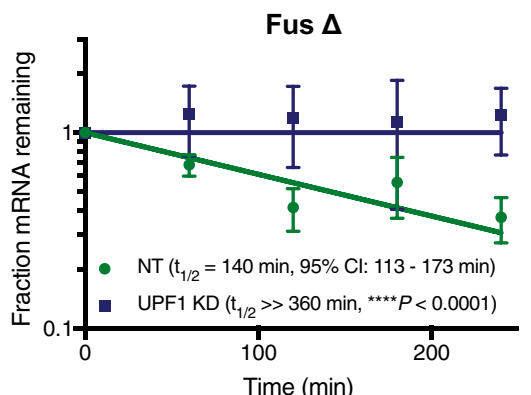
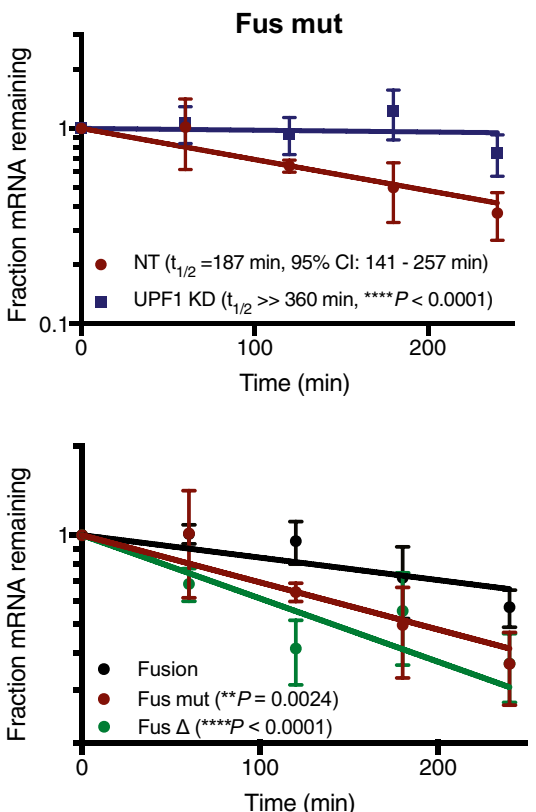

Figure 5. hnRNP L protects the BCL2 fusion transcript from NMD.

A Schematic of normal BCL2 transcript splicing. Introns are indicated with chevrons. Double slashes indicate segments that have been abridged for simplicity. The light blue region of the $3^{\prime} U T R$ of BCL2 represents its first 400 nucleotides, with the sequence provided below. Nucleotides in red indicate stretches of hnRNP $L$ recognition sequence.

B Schematic of aberrant BCL2:IGH mRNAs generated by the $t(14 ; 18)$ translocation event found in many B cell lymphomas. Dotted chevrons indicate alternative events that generate either the secreted or membrane-bound form of $\mathrm{gH}$.

C qPCR of selected transcripts in SU-DHL-4 cells under conditions of non-targeting RNAi or hnRNP L knockdown with or without $50 \mu \mathrm{g} / \mathrm{ml}$ cycloheximide treatment for $4 \mathrm{~h}(n \geq 3)$. Significance of hnRNP L KD + DMSO and NT + CHX was compared to NT + DMSO, and hnRNP L KD + CHX was compared to hnRNP L KD + DMSO. Bars represent the mean $\pm S D$. For clarity, only a single star was used to indicate significance; in all cases, ${ }^{*} P \leq 0.05$ in two-tailed Student's $t$-tests.

D Schematic of the reporter constructed to assay protection of the fusion transcript, consistent with the color scheme in (B). Spliced sequences are indicated with chevrons (see Appendix Fig S3 for alignments). Bright pink indicates the AdML major-late intron used in place of the long intron between J and the first $\mathrm{C} \gamma$ ICH exon. Only exons encoding secreted IgH were used.

E Half-life determination from RT-qPCR detection of reporter constructs in HeLa Tet-Off cells transfected with non-targeting or UPF1 siRNAs. For plots comparing nontargeting with UPF1 knockdown, significance is compared to the non-targeting condition using the sum-of-squares $F$ test. The fourth plot compares the nontargeting decay curves of the three reporters, and significance is compared to the decay of the fusion transcript. Points represent the mean \pm SD from qRT-PCR at each time point of at least three independent experiments. 
A

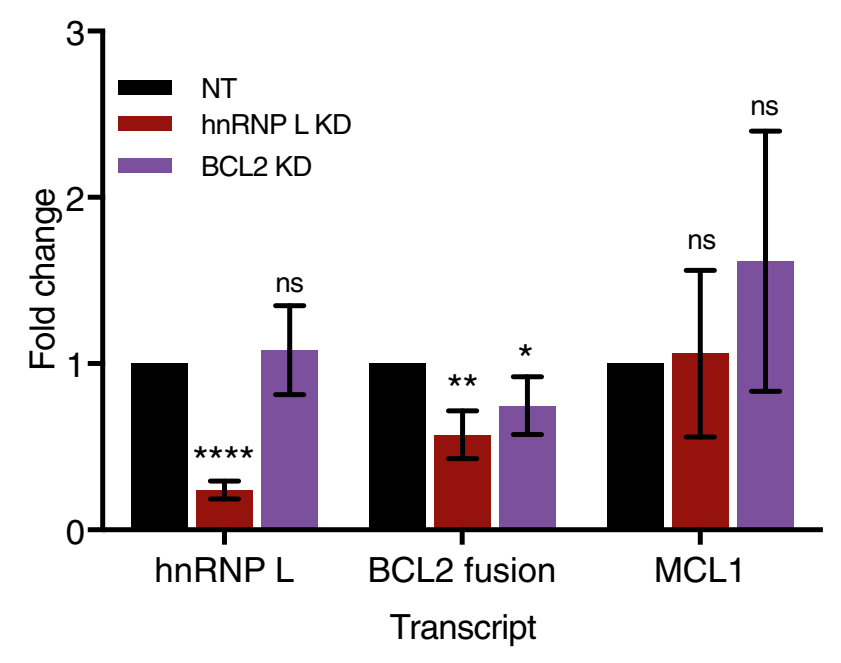

B

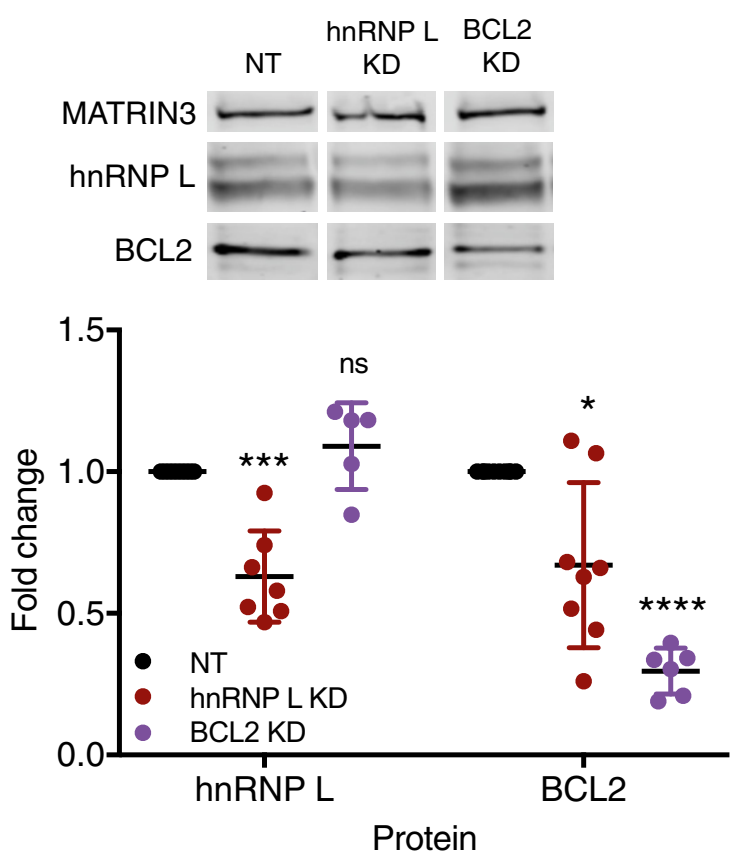

\section{C}
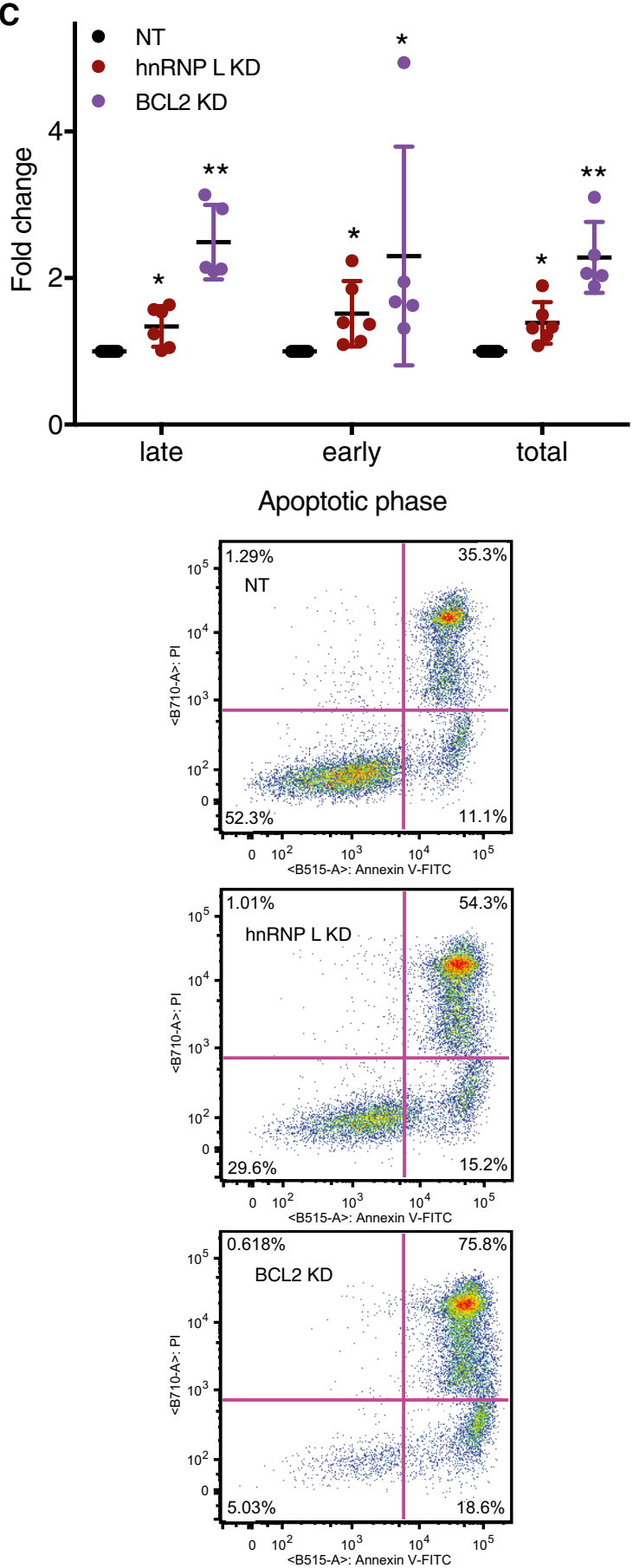

Figure 6. hnRNP L depletion results in increased apoptosis in SU-DHL-4 cells.

A GPCR of selected transcripts in SU-DHL-4 cells under conditions of BCL2 knockdown, hnRNP L knockdown, or non-targeting control siRNA nucleofection ( $n \geq 4$ independent experiments). Bars indicate mean \pm SD. Significance was determined compared to NT using two-tailed Student's $t$-tests.

B Quantification of protein reduction in knockdown experiments and a representative western blot. Each point represents a trial ( $n \geq 5$ for a given condition). Means with standard deviation are indicated on the plot. Target protein levels from all biological replicates were normalized to MATRIN3, with statistical analysis as in panel (A).

C FACS-based apoptosis analysis of SU-DHL-4 cells nucleofected using the indicated siRNAs and exposed to $5 \mu \mathrm{M}$ MCL1 inhibitor for 24 h. Quantification of relative change in each quadrant is provided where points represent individual experiments ( $n \geq 5$ for a given condition). Means with standard deviation are indicated on the plot, and statistical analysis was performed as in panel (A). A representative FACS experiment is shown with the percentage of cells in each gate indicated.

Data information: ${ }^{*} P \leq 0.05,{ }^{* *} P \leq 0.01,{ }^{* * \star} P \leq 0.001,{ }^{* \star *} P \leq 0.0001$.

Source data are available online for this figure. 
hnRNP L knockdown in SU-DHL-4 cells resulted in significant reduction of BCL2:IGH fusion mRNA and BCL2 protein levels (Fig 6A and B). Viability assays using cells treated with increasing MCL1 inhibitor doses indicated that modest hnRNP L knockdown significantly sensitized the cells to the MCL1 inhibitor, consistent with the reduction in BCL2 expression (Fig EV4A). To confirm that cell death upon hnRNP L knockdown was due to apoptosis, we performed flow cytometry on cells exposed to $5 \mu \mathrm{M}$ MCL1 inhibition and stained with Annexin V/propidium iodide. In this assay, the overall proportion of apoptotic cells increased significantly with combined hnRNP L reduction and MCL1 inhibition, as compared to cells treated with non-targeting siRNA and the same dose of MCL1 inhibitor (Fig 6C). The majority of this effect was due to a significant increase in the proportion of cells undergoing early apoptosis. As expected, given that hnRNP L knockdown must result in sequential loss of hnRNP L followed by BCL2 to generate a phenotype, this effect was less pronounced than that caused by the more efficient direct depletion of BCL2 (Figs 6C and EV4A). We also performed knockdown experiments in DoHH2 cells, a distinct lymphoma cell line that carries a variant of the BCL2:IGH fusion and is also resistant to venetoclax due to high MCL1 expression (Kluin-Nelemans et al, 1991; Dyer et al, 1996; Choudhary et al, 2015). Here, too, hnRNP L knockdown sensitized cells to MCL1 inhibition (Fig EV4B and $\mathrm{C}$ ).

\section{CRISPR/Cas9 deletion of hnRNP L binding sites reduces BCL2 expression and activity}

hnRNP L is a multi-functional protein that may impact apoptosis through mechanisms beyond BCL2 protection from NMD. In order to directly assess the role of hnRNP L binding to the BCL2 transcript, we designed guide RNAs to direct selective CRISPR/Cas9-mediated deletion of a 197-nt segment of the BCL2 3'UTR comprising the area of greatest hnRNP L binding potential, including the dense 49-nt stretch of CA repeats (Figs 7A and EV5A). For these experiments, we opted to use SU-DHL-6 cells, which like SU-DHL-4 cells contain a $t(14: 18)$ translocation at the BCL2 MBR but exhibit greater dependence on BCL2. Critically, SU-DHL-6 cells do not use the MCL1mediated compensatory mechanism and are thus significantly more sensitive to BCL2 inhibition by venetoclax than SU-DHL-4 cells, as shown both here and by others (Fig 7B; Souers et al, 2013; Vervloessem et al, 2017). Consistent with our findings in all cell lines studied, transient depletion of hnRNP L in SU-DHL-6 resulted in the significant reduction of the BCL2 fusion transcripts, indicating suitability for further studies of the importance of hnRNP L binding for NMD evasion (Fig 7C).

Nucleofection of Cas9 RNPs complexed with guide RNAs targeting sequences flanking the primary CA-rich tract resulted in approximately 35\% deletion efficiency in SU-DHL-6 cells (Figs 7D and EV5B). Although incomplete deletion resulted in phenotype variability from trial to trial, it was nonetheless sufficient to generate insight into population-level BCL2 expression and apoptosis upon hnRNP L binding site disruption. PCR amplification and sequencing of the TC-proximal region confirmed that the intended 3'UTR segment was accurately deleted, without alteration of the BCL2 coding sequence (Fig EV5C). Despite leaving intact some TC-proximal hnRNP L binding motifs in the edited $3^{\prime} \mathrm{UTR}$, the deletion was sufficient to cause a significant reduction in the levels of both BCL2 fusion mRNAs and protein (Fig 7E and F). Importantly, reduction in BCL2 expression through $3^{\prime}$ UTR editing was accompanied by an increase in apoptosis (Fig 7G). Taken together, the CRISPR data, along with RNAimediated transcript reductions in multiple cell lines, indicate that hnRNP L plays an important role in preventing apoptosis of B cell lymphoma lines through modulation of BCL2 levels.

\section{Discussion}

The increased transcriptome complexity found in metazoans has evolved alongside a continued need for effective mRNA quality control. Correspondingly, the vertebrate NMD machinery has expanded to include additional proteins responsible for regulation,

Figure 7. Deletion of hnRNP L binding site in the BCL2 $3^{\prime}$ UTR results in increased apoptosis in SU-DHL-6 cells.

A Schematic of deleted region in CRISPR/Cas9-edited cells. A portion of the $3^{\prime} U T R$ at the BCL2 locus is represented, with the last exon as a wide box and the $3^{\prime} U T R$ as a narrow box. The light blue region of the $3^{\prime} U T R$ represents its first 400 nucleotides, in which the relevant hnRNP L binding sites are located. The " $X$ " indicates the region of the deletion.

B SU-DHL-4 and SU-DHL-6 cells were treated with varying doses of BCL2 inhibitor. Cell viability was measured after $24 \mathrm{~h}$ of drug treatment using an assay for ATP concentration as a proxy for live cells. Traces were generated from at least five independent biological replicates. Points represent mean \pm SD at each dose. $P$-value from comparison of dose response between SU-DHL-4 and SU-DHL-6 cells calculated using sum-of-squares $F$ test is given in the graph key. Significance of individual points calculated using two-tailed Student's $t$-tests is marked using asterisks on the graph.

C qPCR of selected transcripts in SU-DHL-6 cells under conditions of hnRNP L knockdown or non-targeting control ( $n=5$ independent experiments). Bars indicate mean \pm SD. Significance of hnRNP L KD was determined compared to NT using two-tailed Student's $t$-tests.

D Representative amplification of the region of chromosomal deletion in edited cells. PCR products were run on a $1 \%$ agarose gel to resolve the amplicons from edited and unedited chromosomes.

E qPCR of selected transcripts in edited SU-DHL-6 cells ( $n=4$ independent experiments). Significance was determined as compared to control, and data are represented as in (C).

F Quantification of protein reduction and western blot of lysates from a representative experiment of edited cells. Target protein levels from all biological replicates were normalized to MATRIN3. Each point represents a trial $(n=4)$. Bars indicate mean \pm SD. Significance was determined compared to NT using two-tailed Student's t-tests.

G FACS-based apoptosis analysis of edited SU-DHL-6. Quantification of relative change in each quadrant is provided, where points represent individual experiments ( $n=6$ for a given condition). Means with standard deviation are indicated on the plot, analyzed as in (F). Shown is a representative FACS experiment. Percentage of cells in each gate is indicated.

Data information: ${ }^{*} P \leq 0.05,{ }^{* *} P \leq 0.01,{ }^{* \star \star} P \leq 0.001$

Source data are available online for this figure. 
target identification, and degradation (Baird et al, 2018; Nasif et al, 2018). Complementing this suite of effectors, we propose that sequence-specific inhibitors of NMD are essential for accurate performance of both its regulatory and quality control functions in the context of a complex transcriptome. Our data suggest that PTBP1 and hnRNP L together shield a sizeable proportion of human
A

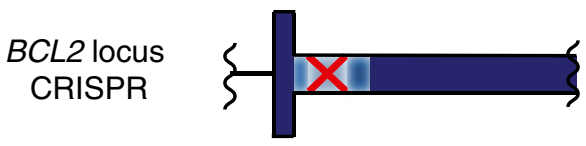

C

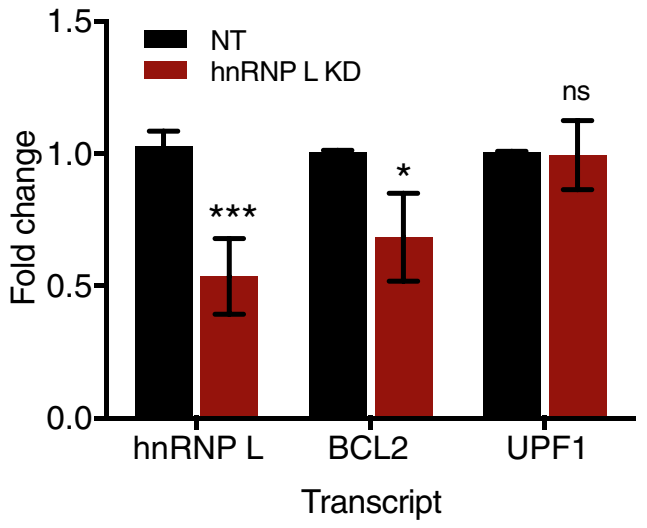

E

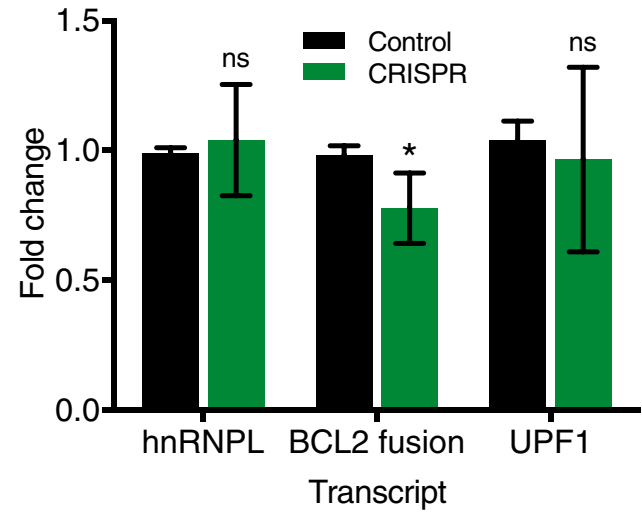

G

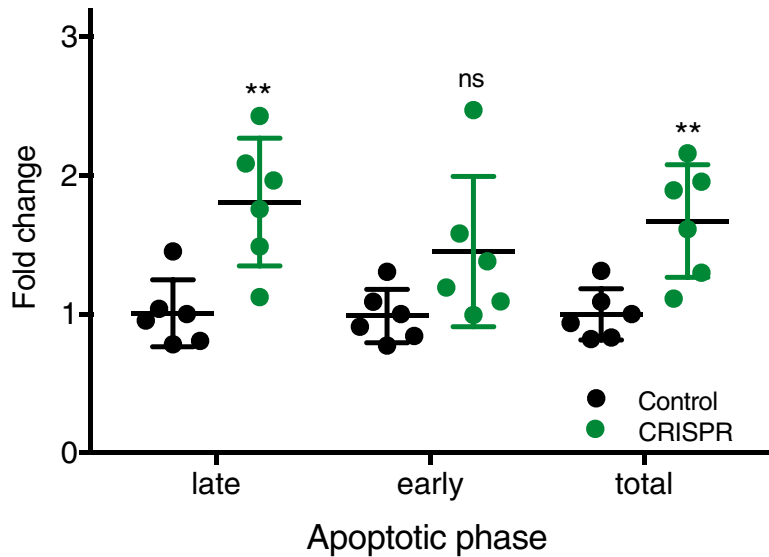

B

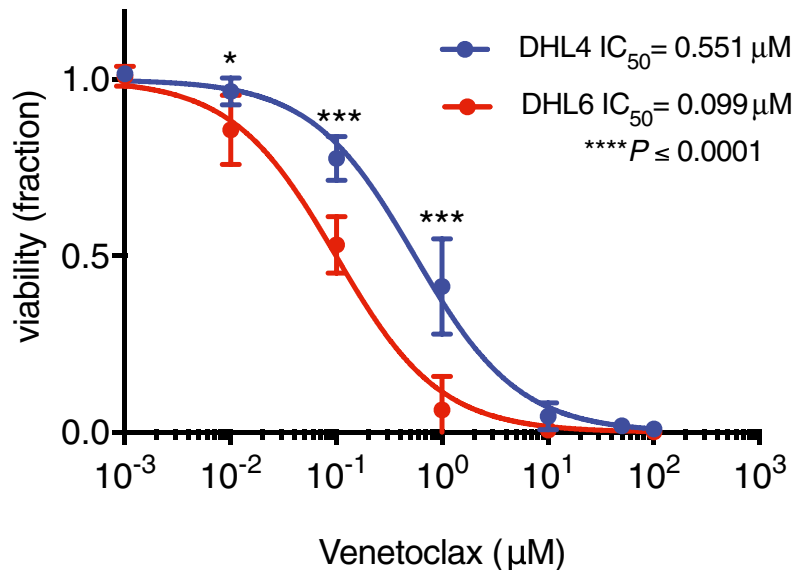

D

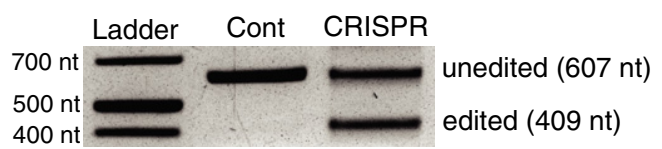

\section{$\mathbf{F}$}

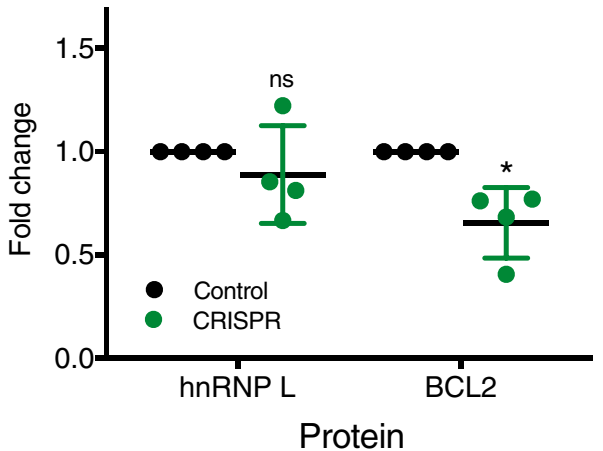

MATRIN3

Cont CRISPR hnRNP L

BCL2
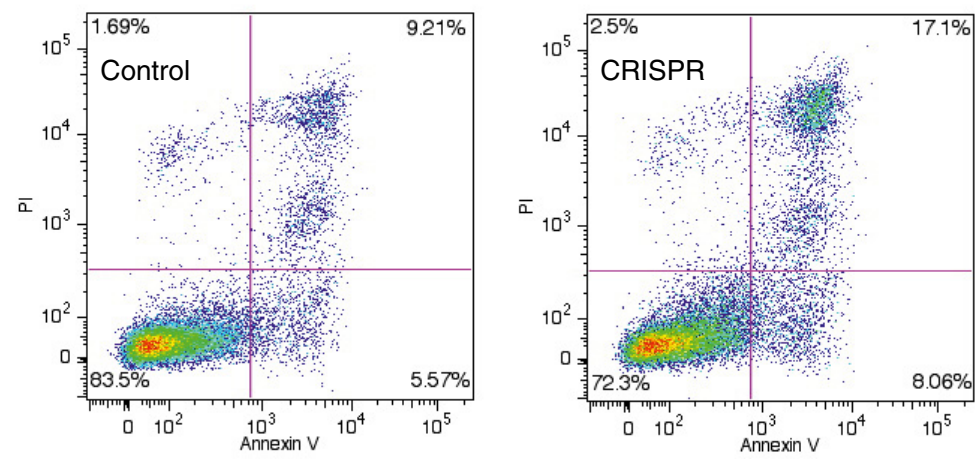

Figure 7. 
mRNAs. Our analysis of the relationship between RNA-binding protein motifs and UPF1 association (Fig 1A) also suggests that there may be additional factors that have an effect on NMD substrate selection and are candidates for future exploration.

Importantly, widespread protection of long 3'UTRs by hnRNP L and PTBP1 may partially explain discrepancies between transcriptome-wide and reporter-based analyses of NMD target selection. Specifically, previous genome-wide studies of UPF1 binding and NMD have identified positive correlations between $3^{\prime}$ UTR length and susceptibility to decay, but these findings were not as clear as studies using reporter mRNAs would suggest (Hurt et al, 2013; Kurosaki et al, 2014). Here, however, we show that long $3^{\prime}$ UTRs capable of recruiting protective proteins are indistinguishable from short 3'UTRs with respect to UPF1 binding and decay (Fig 4). Consequently, accounting for hnRNP L and PTBP1 binding potential markedly increases the power of $3^{\prime} \mathrm{UTR}$ length to predict targets of NMD.

Our studies indicate that the strength of the protective mechanism on a particular transcript can be modulated by varying the number and/or positioning of hnRNP L binding sites (Fig 3C). $3^{\prime}$ UTRs may be programmed for recruitment of protective factors in such a way as to provide a spectrum of stability. Based on our previous work, this feature is shared by PTBP1-mediated protection (Ge et al, 2016). Consistent with our findings from reporter mRNA mutagenesis, transcriptome-wide analysis of the relationship between hnRNP L/PTBP1 motifs and decay susceptibility indicates that transcripts with increased binding potential are more likely to exclude UPF1 and evade NMD than those with fewer motifs. As a result, the degree of NMD evasion may be regulated based both on $3^{\prime}$ UTR sequence as well as hnRNP L availability. Together, these observations imply that many mRNAs exist on a continuum between NMD sensitivity and insensitivity.

Mutagenesis of CSRP1 and BCL2:IGH fusion reporter mRNAs, along with hnRNP L eCLIP analysis, suggest that hnRNP L binding near TCs is particularly important for protection from NMD (Figs 1B, 3C, and 5D). These findings are in agreement with previous studies of the PTBP1-dependent RSE element and genome-wide analysis of PTBP1 binding (Weil \& Beemon, 2006; Ge et al, 2016). However, our data indicate that binding to additional sites in the $3^{\prime}$ UTR may augment the effects of TC-proximal binding. In the ENCODE hnRNP L eCLIP datasets analyzed, the majority of transcripts with peaks in the first 100 nt downstream of the TC also had one or more peaks in the remainder of the $3^{\prime}$ UTR (Fig EV1C and D). Peaks in any given interval outside of the TC-proximal region were insufficient to provide significant protection, but in aggregate were associated with resistance to UPF1 (Figs 1B and EV1C). These findings could in part be due to a bias toward more highly expressed transcripts in CLIP analyses, but are supported by our finding that transcripts with binding sites for each protective protein near the TC are more likely to have additional 3'UTR motifs for that protein than those without (Fig EV2A-D). In sum, these findings suggest that PTBP1 and hnRNP L binding near TCs is the primary determinant of NMD evasion but that association with multiple $3^{\prime} \mathrm{UTR}$ sites may help to antagonize UPF1 binding and decay. These observations are consistent with prior evidence that UPF1 bound along the length of 3'UTRs may participate in decay (Hogg \& Goff, 2010; Kurosaki et al, 2014; Baker \& Hogg, 2017).
Apoptosis plays an indispensable role in development, tissue homeostasis, and tumor suppression. Regulators of apoptosis, such as members of the BCL2 family, must therefore be controlled to appropriately favor cell death or survival in a context-dependent manner (Czabotar et al, 2014). The BCL2 transcript itself is tightly regulated at all levels of gene expression (Schiavone et al, 2000; Cimmino et al, 2005; Heckman et al, 2006; Díaz-Muñoz et al, 2015; Kuwano et al, 2015). The wild-type $\sim 5,000$-nt BCL2 3'UTR incorporates a number of cis-elements for stability and translational control in addition to the hnRNP L binding motifs. In the case of $t(14 ; 18)$ translocations in follicular lymphoma, as many as half of these events occur in a $\sim 290$-bp MBR in the BCL2 3'UTR, a phenomenon not well explained by sequence requirements for double-strand break formation or enhancer function (Tsai et al, 2008; Lieber, 2016). We propose that translocations in this region promote BCL2 expression and cell survival by preserving the antiNMD hnRNP L binding site while removing potentially destabilizing features in the distal half of the $3^{\prime} \mathrm{UTR}$. Notably, translocations in the MBR truncate the BCL2 3'UTR upstream of the miR-15/16 binding site, an event with potential significance in follicular lymphoma based on the established role for loss of BCL2 suppression by miR-15/16 in chronic lymphocytic leukemia (Calin et al, 2002, 2005; Cimmino et al, 2005; Klein et al, 2010). Several additional miRNAs are known to target the BCL2 3'UTR downstream of the MBR, including miR-200bc, miR-181, miR-30, and miR-23 (Chen et al, 2010; Ouyang et al, 2012; Sabirzhanov et al, 2014; Wei et al, 2014; Huang et al, 2015; Wang et al, 2015; Zhong et al, 2016).

The complexity of $3^{\prime}$ UTR-mediated BCL2 regulation illustrates the importance and potential pitfalls of shielding long $3^{\prime}$ UTRs from NMD. In the case of the aberrant BCL2:IGH transcript arising from the $t(14: 18)$ translocation, retention of the hnRNP $\mathrm{L}$ binding element prevents detection by UPF1, eliminating the potential of NMD to rescue BCL2 overexpression. In this way, the protective mechanism, presumably evolved to minimize the effect of NMD on tight 3'UTR-based regulation by other cis-elements, is subverted to allow a high expression level of this pathogenic aberrant transcript.

Beyond the central role of BCL2:IGH fusion mRNAs in the development of many B cell lymphomas, BCL2 overexpression is a common occurrence in numerous additional cancers (Adams \& Cory, 2018). Our data identifying the normal BCL2 $3^{\prime}$ UTR as an NMD target in the absence of hnRNP L suggest that protection from NMD may promote growth of neoplasms overexpressing wild-type BCL2 mRNA. Consistent with this prediction, hnRNP L has been implicated in oncogenesis through promotion of BCL2 expression in multiple tumor types (Zhou et al, 2016; Lv et al, 2017). Recent clinical applications for NMD modulation have centered around NMD inhibition or translational read-through of PTC-containing transcripts in order to mitigate protein deficiencies (Popp \& Maquat, 2016). In contrast, the example of BCL2 provides a case in which increasing transcript sensitivity to NMD may provide therapeutic benefit. Toward this goal, inhibition of hnRNP L itself may be an intriguing avenue for chemotherapy, potentially via blocking protein binding to specific $3^{\prime}$ UTRs with antisense oligonucleotides (Fei et al, 2017; Shen \& Corey, 2018) Additionally, development of compounds for global or transcript-specific NMD enhancement may represent a novel and potentially widely applicable anti-cancer strategy. 


\section{Materials and Methods}

\section{Cultured cells and inhibitors}

HEK-293 Tet-Off (HEK-293TO) and HeLa Tet-Off Advance cells (Clontech, Mountain View, CA) were maintained at $37^{\circ} \mathrm{C}$ in ambient oxygen and 5\% $\mathrm{CO}_{2}$ in DMEM (Gibco \#11965-092) supplemented with $10 \%$ fetal bovine serum (Gibco) and a $1 \%$ penicillin, streptomycin, and L-glutamine mixture (Gibco). SU-DHL-4 (ATCC \#CRL2957), SU-DHL-6 (ATCC \#CRL-2959), and DoHH2 (a kind gift from Louis Staudt, NIH-NCI) were maintained in RPMI-1640 (Gibco \#A10491-01) with the same supplements and incubation conditions. The MCL1 inhibitor A-1210477 (product 21113) and the BCL2 inhibitor ABT-199 (product 16233) were obtained from Cayman Chemical (Ann Arbor, Michigan). Both were resuspended in DMSO to a final concentration of $10 \mathrm{mM}$ and stored at $-80^{\circ} \mathrm{C}$ until use.

\section{Plasmids and siRNAs}

Sequences of all synthetic DNA fragments and oligonucleotides are provided in Appendix Table S2. Construction of pcTET2 $\beta w$ tSMG5 with the RSE and PTB-containing variants was previously described (Ge et al, 2016). The RSE sequence variant in which PTBP1 binding sites were replaced by hnRNP L binding sites was synthesized (Integrated DNA Technologies, Coralville, IA) and inserted into the same backbone. The pcßwtGAP construct was previously described (Singh et al, 2008; Hogg \& Goff, 2010). For CSRP1 reporters, the WT and mutated CSRP1 $3^{\prime}$ UTRs were synthesized (Integrated DNA Technologies, Coralville, IA) and cloned into the pcTET2 $\beta w t \beta$ backbone to allow use of the bGH polyadenylation element (LykkeAndersen et al, 2000). BCL2 fusion reporters with the secreted form of $\mathrm{C} \gamma 1$ were constructed based on the published sequence from $\mathrm{SU}$ DHL-4 cells (Cleary et al, 1986). Segments were assembled by Gibson assembly (NEB, Ipswich, MA) and cloned into the pcTET $2 \beta w t \beta$ backbone. All cloning products were sequence-verified (Eurofins, Louisville, KY). Accession numbers for sequences used to construct BCL2:IGH fusion reporter mRNAs were NM_000633 (BCL2) and NG_001019 (IGH).

siRNAs used were non-targeting siRNA: Silencer Select negative control \#2 siRNA (ThermoFisher Scientific \#4390846); UPF1 siRNA: GAUGCAGUUCCGCUCCAUUUU (ThermoFisher Scientific; Mendell et al, 2004); hnRNP L siRNA used for SU-DHL-4, SU-DHL-6, DoHH2: CGGAUGUUCUUUACACUAUTT (ThermoFisher Scientific \#s6742); hnRNP L siRNA used for HEK-293TO and HeLa Tet-Off: GGCUUGGAU CAAUCUAAGA (ThermoFisher Scientific \#s6740); BCL2 siRNA for SU-DHL-4: GGGAGAACAGGGUACGAUA (Dharmacon \#J-003307-160002); and BCL2 siRNA for SU-DHL-6: GGAUGACUGAGUACCUGAA (ThermoFisher Scientific \#s1916).

\section{Statistical analysis}

All statistical tests were performed using Prism 7 software (GraphPad). All two-tailed Student's t-tests were performed under assumptions of unpaired, parametric datasets. For all experimental datasets, $F$ tests were performed to determine whether variance was shared. If not, Welch's correction was used. For genome-wide data, nonparametric tests were used as indicated in the figure legends.
Unless otherwise noted, we use the following significance convention: $* P \leq 0.05, * * P \leq 0.01, * * * P \leq 0.001, * * * * P \leq 0.0001$, and "ns" is not significant.

\section{Motif analysis}

Position-specific scoring matrices corresponding to the indicated RBP motifs were downloaded from the RBPmap database (Paz et al, 2014). HOMER software was used to search hg19 for motif occurrences (Heinz et al, 2010), and BEDTools was used to identify motifs occurring in 5'UTRs, coding sequences, and 3'UTRs annotated using TACO and IsoformSwitchAnalyzeR software (Quinlan \& Hall, 2010; Niknafs et al, 2017; Vitting-Seerup \& Sandelin, 2017). Transcripts were categorized as having zero, one, or more than one motif in the first $400 \mathrm{nt}$ of the $3^{\prime} \mathrm{UTR}$ for initial analysis of relative recovery in UPF1 RIP-seq (Fig 1A; GEO Series GSE69585; Lee et al, 2015) or by motif density in the $3^{\prime}$ UTR (Fig 4). Box and whisker plots and CDF plots representing motif analysis data were generated by Prism 7 (GraphPad). Statistical significance was determined by nonparametric Kruskal-Wallis tests with Dunn's correction for multiple comparisons. CDF plots represent exact cumulative frequency.

\section{RNA-seq}

Total RNA $(1 \mu \mathrm{g})$ from HEK-293TO cells transfected with the indicated siRNAs was used for library preparation (Ge et al, 2016). Following removal of ribosomal RNA with the Ribo-Zero ${ }^{\mathrm{TM}}$ rRNA Removal Kit (Human/Mouse/Rat; Epicentre), RNA was fragmented and used for library construction with the Illumina TruSeq Stranded Total RNA kit. cDNA libraries were validated using an Illumina MiSeq, and 50-bp paired-end strand-specific sequencing was performed on an Illumina HiSeq 2000. Reads were aligned to the human reference genome hg19 with TopHat2, and differential expression analysis was conducted using DESeq2 (Kim et al, 2013; Love et al, 2014). Significance was adjusted for multiple comparisons. CDF plots representing these data were generated by Prism 7 (GraphPad) and represent exact cumulative frequency. Statistical significance was determined by unpaired, two-tailed Kolmogorov-Smirnov tests or unpaired, two-tailed Kruskal-Wallis tests with Dunn's correction for multiple comparisons, as indicated. Sequencing data are available from the NCBI GEO database, accession GSE109143.

\section{eCLIP analysis}

Peak locations from hnRNP L eCLIP in HepG2 (ENCSR724RDN) and K562 (ENCSR795CAI) cells were downloaded from the ENCODE database (Sundararaman et al, 2016). BEDTools (Quinlan \& Hall, 2010) was used to determine the positions of peak $5^{\prime}$ boundaries relative to TCs annotated as described above. RNASeq reads from K562 cells stably expressing control (GEO Accession GSE88083) or UPF1 (GSE88140) shRNAs were aligned to hg19 using HISAT2, using the index provided by the authors (Kim et al, 2015; Sundararaman et al, 2016), and differential gene expression analysis was performed with DESeq2 (Love et al, 2014). 


\section{RNP-IP}

HEK-293 Tet-Off cells were transfected using TurboFect according to the manufacturer's instructions (ThermoFisher Scientific) with the indicated plasmids $24 \mathrm{~h}$ after plating. Cells were harvested $48 \mathrm{~h}$ post-transfection, resuspended in hypotonic lysis buffer [HLB; $20 \mathrm{mM}$ HEPES (pH 7.6), $2 \mathrm{mM} \mathrm{MgCl}_{2}, 10 \%$ glycerol, $1 \mathrm{mM}$ DTT, supplemented with protease inhibitors], subjected to freeze-thaw lysis, and extracted in $150 \mathrm{mM} \mathrm{NaCl}$ as described (Hogg \& Collins, 2007a,b). For immunoprecipitation, mouse anti-hnRNP L antibody (Abcam \#ab6106) or non-specific mouse IgG (Millipore \#12-371) pre-bound to protein G Dynabeads (Life Technologies, Carlsbad, CA) was incubated in extracts for $2 \mathrm{~h}$, followed by extensive washing with HLB supplemented with $150 \mathrm{mM} \mathrm{NaCl}$ and $0.1 \% \mathrm{NP}-40$. Bound RNA was eluted from beads in TRIzol reagent (Life Technologies, Carlsbad, CA) and probed by northern blot. Analysis of results was performed by unpaired, two-tailed Student's $t$-test with Holm-Sidak multiple comparisons testing.

\section{Metabolic labeling}

To determine specific mRNA half-life measurements during nontargeting and hnRNP L depletion, HEK-293TO cells were reversetransfected with a gene-specific or non-targeting control siRNA for $72 \mathrm{~h}$. At the end of the depletion, cells were treated with $0.5 \mathrm{mM} \mathrm{5-}$ ethynyl uridine (5-EU) for $60 \mathrm{~min}$. Cells were then immediately harvested and RNA was isolated using TRIzol. In addition, Drosophila S2 cells (ThermoFisher Scientific, Cat. No. R690-07) were cultured at ambient temperature for $24 \mathrm{~h}$ in Sf-900 media (ThermoFisher Scientific, Cat. No. 10967032) containing 0.1 mM 5-EU, and RNA was isolated and processed as with the human cells for use as a spike-in control. To differentiate total and nascent RNA levels in each sample, $3.6 \mu \mathrm{g}$ of sample RNA was combined with $400 \mathrm{ng}$ (10\%) of the spike-in control and partitioned using the Click-iT Nascent RNA Capture Kit (ThermoFisher Scientific, Cat. No. C10365) following the manufacturer's protocol. $0.5 \mu \mathrm{g}$ of biotinylated RNA from each sample was used for "total" cDNA library preparation, with the remaining $3 \mu \mathrm{g}$ of RNA from that sample applied to the Streptavidin T1 magnetic beads for labeled RNA capture and cDNA synthesis. The resulting $50 \mu \mathrm{l}$ cDNA libraries were diluted, and mRNA abundance was determined using qRTPCR as previously described. Nascent RNA recovery was normalized to Drosophila RP49 mRNA levels, and individual half-lives were determined using the equation: $t_{1 / 2}=-t_{L} * \ln (2) / \ln (1 / \mathrm{R})$, where $t_{L}$ is the $5^{\prime} \mathrm{EU}$ labeling time in minutes and $R$ is the abundance in nascent RNA fraction/abundance in total RNA fraction (Haque et al, 2018; Russo et al, 2017).

\section{mRNA decay assays}

For time courses without RNAi, HeLa Tet-Off cells were transfected with the indicated plasmid in $60-\mathrm{mm}$ plates using TurboFect and maintained in DMEM supplemented with $10 \%$ FBS and $5 \mathrm{ng} / \mathrm{ml}$ doxycycline (Sigma, St. Louis, MO). Cells were split into four equal aliquots in 12-well plates $24 \mathrm{~h}$ post-DNA transfection. For decay assays performed with RNAi, HeLa Tet-Off cells were reverse-transfected into 24-well plates with 15 pmol of the indicated siRNAs using Lipofectamine RNAiMAX (Life Technologies, Frederick, MD) according to the manufacturer's recommendations (Baird \& Hogg, 2018). The next day, cells were transfected with reporter constructs using TurboFect and suppressed with doxycycline as above.

Forty-eight hours after DNA transfection, cells were washed with $1 \mathrm{ml}$ PBS and incubated in growth medium supplemented with low tetracycline FBS (Clontech) for $4 \mathrm{~h}$. Transcription was quenched by adding doxycycline to a final concentration of $1 \mu \mathrm{g} / \mathrm{ml}$, and cells were harvested in TRIzol after $30 \mathrm{~min}$ and at the indicated intervals. RNA was quantified from northern blots (below) or qPCR (primers/ probes in Appendix Table S2). Prism 7 software (GraphPad) was used to graph the fraction of remaining RNA at the indicated time points on semilog plots and obtain the rate of RNA decay $\left(k_{\text {decay }}\right)$ by fitting to a semilog regression using the least squares method. The slope of each curve was compared to control using the sum-ofsquares $F$ test, and these parameters were used to calculate $95 \%$ confidence intervals. The half-lives of the mRNAs were calculated using the equation: $t_{1 / 2}=\ln 2 / k_{\text {decay }}$.

\section{Northern blots}

RNA was isolated using TRIzol and resolved on $1.2 \%$ formaldehyde/agarose gels. After transfer, ${ }^{32} \mathrm{P}$-labeled in vitro transcribed (NEB T7 RNA polymerase) probes against $\beta$-globin were used in RSE decay time courses, RNP-IPs, and CSRP1 time courses. Northern blots were imaged on a Typhoon Trio+ scanner, and quantification was performed using Image Studio software (LI-COR). Primers for amplification of probe templates are provided in the primer list.

\section{Immunoblot analysis}

Cells were lysed in RIPA buffer [10 mM Tris- $\mathrm{HCl}$ (pH 8.0), $1 \mathrm{mM}$ EDTA, $0.5 \mathrm{mM}$ EGTA, $150 \mathrm{mM} \mathrm{NaCl}, 1 \% \mathrm{NP}-40,0.1 \%$ sodium deoxycholate, $0.1 \%$ SDS, and Halt Protease Inhibitor Cocktail (Thermo Scientific)] for $10 \mathrm{~min}$ at $4^{\circ} \mathrm{C}$ and centrifuged at $12,000 \times \mathrm{g}$ for $10 \mathrm{~min}$ at $4^{\circ} \mathrm{C}$. Protein concentration was measured using the Pierce $660 \mathrm{~nm}$ Protein Assay Reagent (Thermo Scientific) on an Infinite M200 PRO multimode plate reader (Tecan). Equal amounts of protein were loaded on 4-12\% NuPAGE Novex Bis-Tris protein gels (Thermo Scientific). Samples were resolved and transferred to nitrocellulose membranes in accordance with the manufacturer's protocols. Membranes were blocked for $30 \mathrm{~min}$ at room temperature with Blocking Buffer for Fluorescent Western Blotting (Rockland Immunochemicals, Limerick, PA) and blotted with primary antibodies against MATRIN3 (Bethyl Laboratories, Montgomery Texas \#A300-591A 1:1,000), hnRNP L (Abcam ab6106 1:1,000), and BCL2 (for experiments in SU-DHL-4, Cell Signaling Technology 34223 1:500; for experiments in SU-DHL-6, Bethyl Laboratories \#A303675A 1:1,000). Fluorescently labeled secondary antibodies (Thermo Scientific) were used at 1:10,000, and membranes were analyzed on an Odyssey Imaging System (LI-COR). Quantification was performed using Image Studio software (LI-COR).

\section{Nucleofection and cycloheximide treatment of B cell lymphoma lines}

SU-DHL-4 and DoHH2 were nucleofected with siRNA in Amaxa Cell Line Nucleofector Kit V (Lonza, Germany) at setting O-17 with 
5 million cells and 200 pmol siRNA per reaction. For RNAi experiments, SU-DHL-6 were nucleofected at setting O-07 under identical reaction conditions. After nucleofection in $100 \mu \mathrm{l}$ of Buffer V, cells were plated in $9 \mathrm{ml}$ of the appropriate regular growth media.

The double knockdown of UPF1 and hnRNP L in SU-DHL-4 cells resulted in dramatically decreased cell viability, and thus, cycloheximide was used for NMD inhibition (EMD Millipore, Billerica, MA, resuspended in DMSO to stock concentration of $10 \mathrm{mg} / \mathrm{ml}$ ). Approximately 500,000 of the indicated nucleofected cells were treated with a final concentration of $50 \mu \mathrm{g}$ cycloheximide/ml media for $4 \mathrm{~h}$ at $37^{\circ} \mathrm{C}$.

\section{CRISPR and validation}

Gene editing of SU-DHL-6 cells was performed according to the Alt$\mathrm{R}^{\circledR}$ CRISPR-Cas9 system guidelines (IDT Coralville, IA). Guide RNA design of sequences targeting the proximal BCL2 $3^{\prime}$ UTR was performed using IDT software, and the sequences predicted to be the highest efficiency with the lowest chance for off-target cleavage were selected. The upstream gRNA sequence was GUACCAUGAAA CAAAGCUGC on the forward strand, and the downstream sequence was GUGGUCGGAUUUCCAAAGAC on the reverse strand. Negative control gRNA (negative control $\# 1$, product 1072544), tracrRNA (product 1075927), electroporation enhancer (product 1075916), and Alt- $\mathrm{R}^{\circledR}$ S.p. Cas9 nuclease (product 1081058) for the system were purchased from IDT. Briefly, each guide RNA and tracrRNA was duplexed by heating to $95^{\circ} \mathrm{C}$ and slow cooling in a $1: 1$ molar ratio. RNP complexes were made with a 2:1 molar ratio of duplexed RNA to Cas9 and scaled in order to have a final quantity of $2.5 \mu \mathrm{M}$ RNP in the electroporation reaction. RNP reaction components were incubated together at room temperature for 20 min prior to use. Two million SU-DHL-6 cells were resuspended in $75 \mu \mathrm{l}$ Buffer $\mathrm{V}$ (Lonza), to which a fixed dose of $4.4 \mu \mathrm{M}$ electroporation enhancer was added along with the RNP complexes. For deletion lines, upstream and downstream guide RNP complexes were combined in a 1:1 ratio. For control lines, RNPs with the single guide were used. Cells were nucleofected with the O-07 setting and allowed to recover as above. Fluorescence-activated cell sorting (FACS) experiments were performed after a minimum of $72 \mathrm{~h}$ of recovery, and cells were passaged prior to each subsequent experiment. The editing efficiency was monitored by RT-PCR with each passage.

Confirmation of editing was performed using PCR with primers flanking the edited region (primers listed in Appendix Table S2). Reactions contained $3 \%$ DMSO and were annealed at $62^{\circ} \mathrm{C}$. Input genomic DNA was extracted from CRISPR lines using Quick-DNA ${ }^{\mathrm{TM}}$ Miniprep Plus Kit (Zymo Research Irvine, California, D4068). Resulting amplicons were separated via electrophoresis on a $1 \%$ agarose gel and measured using the GeneRuler $1 \mathrm{~kb}$ Plus DNA ladder (Thermo Scientific, SM1331). Bands representing edited (409 nt) and unedited (607 nt) chromosomes were individually gel extracted using the MinElute ${ }^{\mathrm{TM}}$ Gel Extraction Kit (Qiagen, 28606) and sent for sequencing using the amplification primers (MacrogenUSA, Rockville, MD).

In order to determine relative amounts of edited and unedited chromosomes, samples were removed from PCRs at 17, 18, 19, and 20 cycles and run on a Novex ${ }^{\mathrm{TM}} 4-20 \%$ TBE gels (Thermo Scientific) as per the manufacturer's recommendation. Gels were stained in $1 \times$
SYBR $^{\mathrm{TM}}$ Gold (Thermo Scientific, S11494) solution for $20 \mathrm{~min}$ and imaged on the Bio-Rad ChemiDoc ${ }^{\mathrm{TM}}$ MP Imaging system with Image Lab 4.1 software. Quantification of bands was performed using Image Studio software (LI-COR).

\section{Flow cytometry}

After $48 \mathrm{~h}$ of recovery from nucleofection, SU-DHL-4 cells were treated with MCL-1 inhibitor for $24 \mathrm{~h}$. After treatment, or $72 \mathrm{~h}$ after nucleofection with RNAi in the case of SU-DHL-6 cells, approximately 500,000 cells were stained using the FITC Annexin V/Dead Cell Apoptosis Kit (Molecular Probes, Eugene, OR) according to the manufacturer's protocol, with the modification that $3 \mu$ l of FITCAnnexin V stain was used. For CRISPR lines, cells were analyzed using the same staining protocol $72 \mathrm{~h}$ after each passage. A total of 20,000 stained cells from each condition were analyzed using the BD LSRFortessa (BD Biosciences), and data were analyzed using FlowJo 9.9.6 (FlowJo, LLC). Gating was performed using the distribution of populations in the NT sample of each replicate. Cell population comparisons were performed using percent of cells in each quadrant, and significance was determined by unpaired two-tailed Student's $t$-tests.

\section{Viability assays}

A total of 10,000 cells of each indicated type were exposed to the indicated concentrations of inhibitor in a total volume of $50 \mu \mathrm{l} \mathrm{stan-}$ dard growth media in each well of a 96-well plate. After $24 \mathrm{~h}$ of exposure, the cells were transferred to a luminometer-compatible plate, and $50 \mu \mathrm{l}$ of CellTiter-Glo Luminescent Cell Viability Assay (Promega, Madison, WI) reagent was added to each well according to the manufacturer's protocol. Luminescence was measured in a Tecan Infinite M200 Pro plate reader. IC $_{50}$ was calculated with Prism 7 software (GraphPad) dose-response 4-parameter fit with variable slope using the least squares method. Significance of $\mathrm{IC}_{50}$ values was calculated using sum-of-squares $F$ test.

\section{qPCR}

RNA was extracted using the TRIzol reagent and treated with RQ1 DNAse (Promega, Madison, WI). 500 ng of each sample was used for cDNA synthesis using the Maxima First Strand cDNA Synthesis Kit for RT-qPCR (Thermo Scientific, Philadelphia, PA). cDNAs were diluted 1:20 with water and used for qPCR with iTaq Universal SYBR Green Supermix (Bio-Rad, Hercules, CA) on a LightCycler 96 thermocycler (Roche, Basel, Switzerland). Relative transcript abundances were calculated by the $\Delta \Delta C_{\mathrm{t}}$ method, and statistical significance was assessed by two-tailed Student's $t$-test (Prism 7 software, GraphPad). The cDNA abundance in each biological replicate was assessed at least twice to minimize technical variation. All primers are listed in the supplement.

For BCL2 reporter decay time courses, time points were extracted in TRIzol and treated with RQ1 DNase for $30 \mathrm{~min}$ at $37^{\circ} \mathrm{C}$. Samples were subsequently cleaned by phenol-chloroform extraction and then treated for $4 \mathrm{~min}$ at $37^{\circ} \mathrm{C}$ with shaking with dsDNase (Thermo Scientific, Philadelphia, PA). qPCR was performed using the iTaq Universal One-Step RT-qPCR Kit (Bio-Rad, Hercules, CA) according to the manufacturer's protocol. No multiplexing was used, and each 
sample was probed twice for both the BCL2:IGH reporter construct and the GFP control. The region of the reporter spanning the BCL2 $3^{\prime}$ UTR and IGH exons was amplified from transfected cells and sequenced in order to confirm correct processing of the reporter. For qPCR, primers were sited to flank the AdML intron in the reporter construct, and the resulting amplicon was size-confirmed as part of assay optimization.

Expanded View for this article is available online.

\section{Acknowledgements}

RNA-seq was performed by the NHLBI DNA Sequencing and Genomics Core, and flow cytometry was performed in the NHLBI Flow Cytometry Core. We thank members of the Hogg Lab for helpful suggestions and critical reading of the manuscript and Louis Staudt for providing DoHH2 cells. This work was supported by the Intramural Research Program, National Institutes of Health, National Heart, Lung, and Blood Institute, and utilized the computational resources of the NIH HPC Biowulf cluster (http://hpc.nih.gov).

\section{Author contributions}

AK: RNA-seq data analysis, CSRP1 time courses, immunoprecipitations, BCL2 plasmid design and time courses, SU-DHL-4, SU-DHL-6, and DoHH2 experiments, study design, and manuscript. ZG: RNA-seq sample preparation, CSRP1 plasmid design and time courses, RSE plasmid design and time courses, and study design. JRH: RNA-seq data analysis, motif analysis, study design, and manuscript.

\section{Conflict of interest}

The authors declare that they have no conflict of interest.

\section{References}

Adams JM, Cory S (2018) The BCL-2 arbiters of apoptosis and their growing role as cancer targets. Cell Death Differ 25: 27-36

Baird TD, Cheng KC-C, Chen Y-C, Buehler E, Martin SE, Inglese J, Hogg JR (2018) ICE1 promotes the link between splicing and nonsense-mediated mRNA decay. Elife 7: e33178

Baird TD, Hogg JR (2018) Using tet-off cells and RNAi knockdown to assay mRNA decay. Methods Mol Biol 1720: 161-173

Baker SL, Hogg JR (2017) A system for coordinated analysis of translational readthrough and nonsense-mediated mRNA decay. PLoS One 12: e0173980

Balagopal V, Beemon KL (2017) Rous sarcoma virus RNA stability element inhibits deadenylation of mRNAs with long 3'UTRs. Viruses 9: E204

Blatter M, Dunin-Horkawicz S, Grishina I, Maris C, Thore S, Maier T, Bindereif A, Bujnicki JM, Allain FH-T (2015) The signature of the five-stranded VRRM fold defined by functional, structural and computational analysis of the hnRNP L protein. J Mol Biol 427: 3001-3022

Bray NL, Pimentel H, Melsted P, Pachter L (2016) Near-optimal probabilistic RNA-seq quantification. Nat Biotechnol 34: 525-527

Bühler M, Steiner S, Mohn F, Paillusson A, Mühlemann O (2006) EJCindependent degradation of nonsense immunoglobulin-mu mRNA depends on 3' UTR length. Nat Struct Mol Biol 13: $462-464$

Calin GA, Dumitru CD, Shimizu M, Bichi R, Zupo S, Noch E, Aldler H, Rattan S, Keating M, Rai K, Rassenti L, Kipps T, Negrini M, Bullrich F, Croce CM (2002) Frequent deletions and down-regulation of micro- RNA genes miR15 and miR16 at 13q14 in chronic lymphocytic leukemia. Proc Natl Acad Sci USA 99: 15524-15529
Calin GA, Ferracin M, Cimmino A, Di Leva G, Shimizu M, Wojcik SE, Iorio MV, Visone R, Sever NI, Fabbri M, Iuliano R, Palumbo T, Pichiorri F, Roldo C, Garzon R, Sevignani C, Rassenti L, Alder H, Volinia S, Liu C-C et al (2005) A MicroRNA signature associated with prognosis and progression in chronic lymphocytic leukemia. N Engl J Med 353: 1793-1801

Celik A, Baker R, He F, Jacobson A (2017) High resolution profiling of NMD targets in yeast reveals translational fidelity as a basis for substrate selection. RNA 23: $735-748$

Chakrabarti S, Bonneau F, Schüssler S, Eppinger E, Conti E (2014) Phosphodependent and phospho-independent interactions of the helicase UPF1 with the NMD factors SMG5-SMG7 and SMG6. Nucleic Acids Res 42: 9447-9460

Chen G, Zhu W, Shi D, Lv L, Zhang C, Liu P, Hu W (2010) MicroRNA-181a sensitizes human malignant glioma U87MG cells to radiation by targeting Bcl-2. Oncol Rep 23: 997-1003

Chester A, Somasekaram A, Tzimina M, Jarmuz A, Gisbourne J, O'Keefe R, Scott J, Navaratnam N (2003) The apolipoprotein B mRNA editing complex performs a multifunctional cycle and suppresses nonsense-mediated decay. EMBO J 22: $3971-3982$

Choudhary GS, Al-Harbi S, Mazumder S, Hill BT, Smith MR, Bodo J, Hsi ED, Almasan A (2015) MCL-1 and BCL-xL-dependent resistance to the BCL2 inhibitor ABT-199 can be overcome by preventing PI3K/AKT/mTOR activation in lymphoid malignancies. Cell Death Dis 6: e1593

Cimmino A, Calin GA, Fabbri M, Iorio MV, Ferracin M, Shimizu M, Wojcik SE, Aqeilan RI, Zupo S, Dono M, Rassenti L, Alder H, Volinia S, Liu C-C, Kipps TJ, Negrini M, Croce CM (2005) miR-15 and miR-16 induce apoptosis by targeting BCL2. Proc Natl Acad Sci USA 102: 13944-13949

Cleary ML, Smith SD, Sklar J (1986) Cloning and structural analysis of CDNAs for bcl-2 and a hybrid bcl-2/immunoglobulin transcript resulting from the $t(14 ; 18)$ translocation. Cell 47: 19-28

Czabotar PE, Lessene G, Strasser A, Adams JM (2014) Control of apoptosis by the BCL-2 protein family: implications for physiology and therapy. Nat Rev Mol Cell Biol 15: 49-63

Deng J, Carlson N, Takeyama K, Dal Cin P, Shipp M, Letai A (2007) BH3 profiling identifies three distinct classes of apoptotic blocks to predict response to ABT-737 and conventional chemotherapeutic agents. Cancer Cell 12: 171-185

Díaz-Muñoz MD, Bell SE, Turner M (2015) Deletion of AU-rich elements within the $B C 123^{\prime} U T R$ reduces protein expression and $B$ cell survival in vivo. PLoS One 10: e0116899

Dyer MJ, Lillington DM, Bastard C, Tilly H, Lens D, Heward JM, Stranks C, Morilla R, Monrad S, Guglielmi P, Kluin-Nelemans JC, Hagemeijer A, Young BD, Catovsky D (1996) Concurrent activation of MYC and BCL2 in B cell non-Hodgkin lymphoma cell lines by translocation of both oncogenes to the same immunoglobulin heavy chain locus. Leukemia 10: 1198-1208

Eberle AB, Lykke-Andersen S, Mühlemann O, Jensen TH (2009) SMG6 promotes endonucleolytic cleavage of nonsense mRNA in human cells. Nat Struct Mol Biol 16: 49-55

Fei T, Chen Y, Xiao T, Li W, Cato L, Zhang P, Cotter MB, Bowden M, Lis RT, Zhao SG, Wu Q, Feng FY, Loda M, He HH, Liu XS, Brown M (2017) Genome-wide CRISPR screen identifies HNRNPL as a prostate cancer dependency regulating RNA splicing. Proc Natl Acad Sci USA 114: E5207-E5215

Ge Z, Quek BL, Beemon KL, Hogg JR (2016) Polypyrimidine tract binding protein 1 protects mRNAs from recognition by the nonsense-mediated mRNA decay pathway. Elife 5: e11155

Haque N, Ouda R, Chen C, Ozato K, Robert Hogg J (2018) ZFR coordinates crosstalk between RNA decay and transcription in innate immunity. Nat Commun 9: 1145 
Heckman CA, Duan H, Garcia PB, Boxer LM (2006) Oct transcription factors mediate $\mathrm{t}(14 ; 18)$ lymphoma cell survival by directly regulating bcl-2 expression. Oncogene 25: 888-898

Heinz S, Benner C, Spann N, Bertolino E, Lin YC, Laslo P, Cheng JX, Murre C, Singh H, Glass CK (2010) Simple combinations of lineage-determining transcription factors prime cis-regulatory elements required for macrophage and B cell identities. Mol Cell 38: 576-589

Hogg JR, Collins K (2007a) RNA-based affinity purification reveals 7SK RNPs with distinct composition and regulation. RNA 13: 868-880

Hogg JR, Collins K (2007b) Human Y5 RNA specializes a Ro ribonucleoprotein for $5 S$ ribosomal RNA quality control. Genes Deu 21: 3067-3072

Hogg JR, Goff SP (2010) Upf1 senses 3' UTR length to potentiate mRNA decay. Cell 143: 379-389

Hogg JR (2016) Viral evasion and manipulation of host RNA quality control pathways. J Virol 90: 7010-7018

Huang P, Ye B, Yang Y, Shi J, Zhao H (2015) MicroRNA-181 functions as a tumor suppressor in non-small cell lung cancer (NSCLC) by targeting Bcl2. Tumour Biol 36: 3381-3387

Hui J, Stangl K, Lane WS, Bindereif A (2003) HnRNP L stimulates splicing of the eNOS gene by binding to variable-length CA repeats. Nat Struct Biol 10: $33-37$

Huntzinger E, Kashima I, Fauser M, Saulière J, Izaurralde E (2008) SMG6 is the catalytic endonuclease that cleaves mRNAs containing nonsense codons in metazoan. RNA 14: 2609-2617

Hurt JA, Robertson AD, Burge CB (2013) Global analyses of UPF1 binding and function reveal expanded scope of nonsense-mediated mRNA decay. Cenome Res 23: $1636-1650$

Jan CH, Friedman RC, Ruby JG, Bartel DP (2011) Formation, regulation and evolution of Caenorhabditis elegans 3'UTRs. Nature 469: 97-101

Kashima I, Yamashita A, Izumi N, Kataoka N, Morishita R, Hoshino S, Ohno M, Dreyfuss G, Ohno S (2006) Binding of a novel SMG-1-Upf1-eRF1-eRF3 complex (SURF) to the exon junction complex triggers Upf1 phosphorylation and nonsense-mediated mRNA decay. Genes Deu 20: 355-367

Kim D, Pertea C, Trapnell C, Pimentel H, Kelley R, Salzberg SL (2013) TopHat2: accurate alignment of transcriptomes in the presence of insertions, deletions and gene fusions. Genome Biol 14: R36

Kim D, Langmead B, Salzberg SL (2015) HISAT: a fast spliced aligner with low memory requirements. Nat Methods 12: 357-360

Klanova M, Andera L, Brazina J, Svadlenka J, Benesova S, Soukup J, Prukova D, Vejmelkova D, Jaksa R, Helman K, Vockova P, Lateckova L, Molinsky J, Maswabi BCL, Alam M, Kodet R, Pytlik R, Trneny M, Klener P (2016) Targeting of BCL2 family proteins with ABT-199 and homoharringtonine reveals BCL2- and MCL1-dependent subgroups of diffuse large B-cell lymphoma. Clin Cancer Res 22: 1138-1149

Klein U, Lia M, Crespo M, Siegel R, Shen Q, Mo T, Ambesi-Impiombato A, Califano A, Migliazza A, Bhagat G, Dalla-Favera R (2010) The DLEU2/miR15a/16-1 cluster controls B cell proliferation and its deletion leads to chronic lymphocytic leukemia. Cancer Cell 17: 28-40

Kluin-Nelemans HC, Limpens J, Meerabux J, Beverstock GC, Jansen JH, de Jong D, Kluin PM (1991) A new non-Hodgkin's B-cell line (DoHH2) with a chromosomal translocation t(14;18)(q32;q21). Leukemia 5: 221-224

Kurosaki T, Maquat LE (2013) Rules that govern UPF1 binding to mRNA $3^{\prime}$ UTRs. Proc Natl Acad Sci USA 110: 3357-3362

Kurosaki T, Li W, Hoque M, Popp MW-L, Ermolenko DN, Tian B, Maquat LE (2014) A post-translational regulatory switch on UPF1 controls targeted mRNA degradation. Genes Deu 28: 1900-1916

Kuwano Y, Nishida K, Kajita K, Satake Y, Akaike Y, Fujita K, Kano S, Masuda K, Rokutan K (2015) Transformer $2 \beta$ and miR-204 regulate apoptosis through competitive binding to $3^{\prime}$ UTR of BCL2 mRNA. Cell Death Differ 22: $815-825$

Le Hir H, Saulière J, Wang Z (2016) The exon junction complex as a node of post-transcriptional networks. Nat Reu Mol Cell Biol 17: 41-54

Lee D-H, Lim M-H, Youn D-Y, Jung SE, Ahn YS, Tsujimoto Y, Lee J-H (2009) hnRNP $L$ binds to CA repeats in the $3^{\prime} U T R$ of bcl-2 mRNA. Biochem Biophys Res Commun 382: 583-587

Lee SR, Pratt GA, Martinez FJ, Yeo GW, Lykke-Andersen J (2015) Target discrimination in nonsense-mediated mRNA decay requires Upf1 ATPase activity. Mol Cell 59: 413-425

Leich E, Ott G, Rosenwald A (2011) Pathology, pathogenesis and molecular genetics of follicular NHL. Best Pract Res Clin Haematol 24: 95-109

Li L, Pongtornpipat P, Tiutan T, Kendrick SL, Park S, Persky DO, Rimsza LM, Puvvada SD, Schatz JH (2015) Synergistic induction of apoptosis in highrisk DLBCL by BCL2 inhibition with ABT-199 combined with pharmacologic Ioss of MCL1. Leukemia 29: 1702-1712

Lieber MR (2016) Mechanisms of human lymphoid chromosomal translocations. Nat Reu Cancer 16: 387-398

Lim M-H, Lee D-H, Jung SE, Youn D-Y, Park CS, Lee J-H (2010) Effect of modulation of hnRNP L levels on the decay of bcl-2 mRNA in MCF-7 cells. Korean J Physiol Pharmacol 14: 15-20

Loh B, Jonas S, Izaurralde E (2013) The SMG5-SMG7 heterodimer directly recruits the CCR4-NOT deadenylase complex to mRNAs containing nonsense codons via interaction with POP2. Genes Deu 27: 2125-2138

Losson R, Lacroute $F$ (1979) Interference of nonsense mutations with eukaryotic messenger RNA stability. Proc Natl Acad Sci USA 76: 5134-5137

Love MI, Huber W, Anders S (2014) Moderated estimation of fold change and dispersion for RNA-seq data with DESeq2. Genome Biol 15: 550

Lv D, Wu H, Xing R, Shu F, Lei B, Lei C, Zhou X, Wan B, Yang Y, Zhong L, Mao $X$, Zou Y (2017) HnRNP-L mediates bladder cancer progression by inhibiting apoptotic signaling and enhancing MAPK signaling pathways. Oncotarget 8: 13586-13599

Lykke-Andersen J, Shu MD, Steitz JA (2000) Human Upf proteins target an mRNA for nonsense-mediated decay when bound downstream of a termination codon. Cell 103: 1121-1131

Maquat LE, Kinniburgh AJ, Rachmilewitz EA, Ross J (1981) Unstable betaglobin mRNA in mRNA-deficient beta o thalassemia. Cell 27: 543-553

Martinez-Contreras R, Cloutier P, Shkreta L, Fisette J-F, Revil T, Chabot B (2007) hnRNP proteins and splicing control. Adu Exp Med Biol 623: $123-147$

Matsuda D, Hosoda N, Kim YK, Maquat LE (2007) Failsafe nonsense-mediated mRNA decay does not detectably target elF4E-bound mRNA. Nat Struct Mol Biol 14: 974-979

Mayr C, Bartel DP (2009) Widespread shortening of 3'UTRs by alternative cleavage and polyadenylation activates oncogenes in cancer cells. Cell 138 673-684

Mendell JT, Sharifi NA, Meyers JL, Martinez-Murillo F, Dietz HC (2004) Nonsense surveillance regulates expression of diverse classes of mammalian transcripts and mutes genomic noise. Nat Genet 36: 1073-1078

Nasif S, Contu L, Mühlemann O (2018) Beyond quality control: the role of nonsense-mediated mRNA decay (NMD) in regulating gene expression. Semin Cell Deu Biol 75: 78-87

Neu-Yilik G, Raimondeau E, Eliseev B, Yeramala L, Amthor B, Deniaud A, Huard K, Kerschgens K, Hentze MW, Schaffitzel C, Kulozik AE (2017) Dual function of UPF3B in early and late translation termination. EMBO J 36: 2968-2986

Nicholson P, Josi C, Kurosawa H, Yamashita A, Mühlemann O (2014) A novel phosphorylation-independent interaction between SMG6 and UPF1 is essential for human NMD. Nucleic Acids Res 42: 9217-9235 
Niknafs YS, Pandian B, Iyer HK, Chinnaiyan AM, Iyer MK (2017) TACO produces robust multisample transcriptome assemblies from RNA-seq. Nat Methods 14: $68-70$

Oberstrass FC, Auweter SD, Erat M, Hargous Y, Henning A, Wenter P, Reymond L, Amir-Ahmady B, Pitsch S, Black DL, Allain FH-T (2005) Structure of PTB bound to RNA: specific binding and implications for splicing regulation. Science 309: 2054-2057

Okada-Katsuhata Y, Yamashita A, Kutsuzawa K, Izumi N, Hirahara F, Ohno S (2012) N- and C-terminal Upf1 phosphorylations create binding platforms for SMG-6 and SMG-5:SMG-7 during NMD. Nucleic Acids Res 40: 1251-1266

Ouyang Y-B, Lu Y, Yue S, Giffard RG (2012) miR-181 targets multiple Bcl-2 family members and influences apoptosis and mitochondrial function in astrocytes. Mitochondrion 12: 213-219

Paz I, Kosti I, Ares M Jr, Cline M, Mandel-Gutfreund Y (2014) RBPmap: a web server for mapping binding sites of RNA-binding proteins. Nucleic Acids Res 42: W361-W367

Peltz SW, Brown AH, Jacobson A (1993) mRNA destabilization triggered by premature translational termination depends on at least three cis-acting sequence elements and one trans-acting factor. Cenes Dev 7: 1737-1754

Phillips DC, Xiao Y, Lam LT, Litvinovich E, Roberts-Rapp L, Souers AJ, Leverson JD (2016) Loss in MCL-1 function sensitizes non-Hodgkin's lymphoma cell lines to the BCL-2-selective inhibitor venetoclax (ABT-199). Blood Cancer J 6: e403

Popp MW, Maquat LE (2016) Leveraging rules of nonsense-mediated mRNA decay for genome engineering and personalized medicine. Cell 165: 1319-1322

Quinlan AR, Hall IM (2010) BEDTools: a flexible suite of utilities for comparing genomic features. Bioinformatics 26: 841-842

Rebbapragada I, Lykke-Andersen J (2009) Execution of nonsense-mediated mRNA decay: what defines a substrate? Curr Opin Cell Biol 21: 394-402

Russo J, Heck AM, Wilusz J, Wilusz CJ (2017) Metabolic labeling and recovery of nascent RNA to accurately quantify mRNA stability. Methods 120 : $39-48$

Sabirzhanov B, Zhao Z, Stoica BA, Loane DJ, Wu J, Borroto C, Dorsey SC, Faden Al (2014) Downregulation of miR-23a and miR-27a following experimental traumatic brain injury induces neuronal cell death through activation of proapoptotic Bcl-2 proteins. J Neurosci 34: 10055-10071

Schiavone N, Rosini P, Quattrone A, Donnini M, Lapucci A, Citti L, Bevilacqua A, Nicolin A, Capaccioli S (2000) A conserved AU-rich element in the $3^{\prime}$ untranslated region of bcl-2 mRNA is endowed with a destabilizing function that is involved in bcl-2 down-regulation during apoptosis. FASEB J 14: $174-184$

Schuetz JM, Johnson NA, Morin RD, Scott DW, Tan K, Ben-Nierah S, Boyle M, Slack GW, Marra MA, Connors JM, Brooks-Wilson AR, Gascoyne RD (2012) BCL2 mutations in diffuse large B-cell lymphoma. Leukemia 26: $1383-1390$

Schüler F, Dölken L, Hirt C, Kiefer T, Berg T, Fusch G, Weitmann K, Hoffmann W, Fusch C, Janz S, Rabkin CS, Dölken G (2009) Prevalence and frequency of circulating $\mathrm{t}(14 ; 18)-\mathrm{MBR}$ translocation carrying cells in healthy individuals. Int J Cancer 124: 958-963

Seto M (2002) Molecular mechanisms of lymphomagenesis through transcriptional disregulation by chromosome translocation. Int J Hematol 76(Suppl 1): $323-326$

Shen X, Corey DR (2018) Chemistry, mechanism and clinical status of antisense oligonucleotides and duplex RNAs. Nucleic Acids Res 46: 1584-1600

Singh G, Rebbapragada I, Lykke-Andersen J (2008) A competition between stimulators and antagonists of Upf complex recruitment governs human nonsense-mediated mRNA decay. PLoS Biol 6: e111
Souers AJ, Leverson JD, Boghaert ER, Ackler SL, Catron ND, Chen J, Dayton BD, Ding H, Enschede SH, Fairbrother WJ, Huang DCS, Hymowitz SG, Jin S, Khaw SL, Kovar PJ, Lam LT, Lee J, Maecker HL, Marsh KC, Mason KD et al (2013) ABT-199, a potent and selective BCL-2 inhibitor, achieves antitumor activity while sparing platelets. Nat Med 19: 202-208

Sundararaman B, Zhan L, Blue SM, Stanton R, Elkins K, Olson S, Wei X, Van Nostrand EL, Pratt GA, Huelga SC, Smalec BM, Wang X, Hong EL, Davidson JM, Lécuyer E, Graveley BR, Yeo GW (2016) Resources for the comprehensive discovery of functional RNA elements. Mol Cell 61: 903-913

Toma KG, Rebbapragada I, Durand S, Lykke-Andersen J (2015) Identification of elements in human long $3^{\prime}$ UTRs that inhibit nonsense-mediated decay. RNA 21: 887-897

Tsai AG, Lu H, Raghavan SC, Muschen M, Hsieh C-L, Lieber MR (2008) Human chromosomal translocations at $\mathrm{CpG}$ sites and a theoretical basis for their lineage and stage specificity. Cell 135: 1130-1142

Van Nostrand EL, Pratt GA, Shishkin AA, Gelboin-Burkhart C, Fang MY, Sundararaman B, Blue SM, Nguyen TB, Surka C, Elkins K, Stanton R, Rigo F, Guttman M, Yeo GW (2016) Robust transcriptome-wide discovery of RNA-binding protein binding sites with enhanced CLIP (eCLIP). Nat Methods 13: 508-514

Vervloessem T, Akl H, Tousseyn T, De Smedt H, Parys JB, Bultynck G (2017) Reciprocal sensitivity of diffuse large B-cell lymphoma cells to BCl-2 inhibitors BIRD-2 versus venetoclax. Oncotarget 8: 111656-111671

Vitting-Seerup K, Sandelin A (2017) The landscape of isoform switches in human cancers. Mol Cancer Res 15: 1206-1220

Wang H, Li J, Chi H, Zhang F, Zhu X, Cai J, Yang X (2015) MicroRNA-181C targets $\mathrm{BCl}-2$ and regulates mitochondrial morphology in myocardial cells. J Cell Mol Med 19: 2084-2097

Wei C, Li L, Gupta S (2014) NF-kB-mediated miR-30b regulation in cardiomyocytes cell death by targeting $\mathrm{BCl}-2$. Mol Cell Biochem 387: $135-141$

Weil JE, Beemon KL (2006) A $3^{\prime}$ UTR sequence stabilizes termination codons in the unspliced RNA of Rous sarcoma virus. RNA 12: 102-110

Weil JE, Hadjithomas M, Beemon KL (2009) Structural characterization of the Rous sarcoma virus RNA stability element. J Virol 83: 2119-2129

Withers JB, Beemon KL (2010) Structural features in the Rous sarcoma virus RNA stability element are necessary for sensing the correct termination codon. Retrovirology 7: 65

Yepiskoposyan $\mathrm{H}$, Aeschimann F, Nilsson D, Okoniewski M, Muhlemann O (2011) Autoregulation of the nonsense-mediated mRNA decay pathway in human cells. RNA 17: $2108-2118$

Zhang W, Zeng F, Liu Y, Zhao Y, Lv H, Niu L, Teng M, Li X (2013) Crystal structures and RNA-binding properties of the RNA recognition motifs of heterogeneous nuclear ribonucleoprotein L: insights into its roles in alternative splicing regulation. J Biol Chem 288: 22636-22649

Zhong X, Zheng L, Shen J, Zhang D, Xiong M, Zhang Y, He X, Tanyi JL, Yang F, Montone KT, Chen X, Xu C, Xiang AP, Huang Q, Xu X, Zhang L (2016) Suppression of MicroRNA 200 family expression by oncogenic KRAS activation promotes cell Survival and epithelial-mesenchymal transition in KRAS-driven cancer. Mol Cell Biol 36: 2742-2754

Zhou X, Li Q, He J, Zhong L, Shu F, Xing R, Lv D, Lei B, Wan B, Yang Y, Wu H, Mao X, Zou $Y$ (2016) HnRNP-L promotes prostate cancer progression by enhancing cell cycling and inhibiting apoptosis. Oncotarget 8 : $19342-19353$

Zünd D, Gruber AR, Zavolan M, Mühlemann O (2013) Translation-dependent displacement of UPF1 from coding sequences causes its enrichment in $3^{\prime}$ UTRs. Nat Struct Mol Biol 20: 936-943 